Korean J. Comput. \& Appl. Math. Vol. 9 (2002), No. 2, pp. 289-347

INVITED ARTICLE

\title{
INTRODUCTION TO DIFFUSIVE LOGISTIC EQUATIONS IN POPULATION DYNAMICS
}

\author{
KAZUAKI TAIRA \\ Dedicated to Professor Izumi Kubo on the occasion of his 60th birthday
}

\begin{abstract}
The purpose of this paper is to provide a careful and accessible exposition of diffusive logistic equations with indefinite weights which model population dynamics in environments with strong spatial heterogeneity. We prove that the most favorable situations will occur if there is a relatively large favorable region (with good resources and without crowding effects) located some distance away from the boundary of the environment. Moreover, we prove that a population will grow exponentially until limited by lack of available resources if the diffusion rate is below some critical value; this idea is generally credited to Thomas Malthus. On the other hand, if the diffusion rate is above this critical value, then the model obeys the logistic equation introduced by P. F. Verhulst.
\end{abstract}

AMS Mathematics Subject Classification : 35J65, 35P30, 92D25

Key words and phrases: Diffusive logistic equation, population dynamics

\section{Table of Contents}

1. Introduction and Main Results

2. Proof of Theorem $1.2-(1)-$

3. Proof of Theorem $1.2-(2)-$

4. Proof of Theorem $1.2-(3)-$

5. Proof of Theorem $1.2-(4)-$

6. Proof of Theorem 1.3

7. The Neumann Case

Appendix: Proof of Remark 1.3

References

Received February 19, 2002.

(c) 2002 Korean Society for Computational \& Applied Mathematics and Korean SIGCAM. 


\section{Introduction and main results}

The purpose of this paper is to illustrate how the theory of linear elliptic eigenvalue problems with indefinite weight functions can be used to analyze reactiondiffusion models in mathematical ecology and population genetics. The two main components of the models we consider are the "reaction" or growth terms and the "diffusion" or dispersal terms. More precisely, reaction-diffusion equations arise in ecological modeling when nonlinear dynamics describing the growth or decline of a population are combined with a diffusion process describing the spatial dispersal of that population. Solutions to the reaction-diffusion models typically represent population densities, or in related problems of population genetics, the distribution of certain alleles within a population. If the population being modeled can disperse through its environment, then the population density need not be uniform. Assuming that dispersal takes place via random walks or Brownian motion leads to a diffusion equation for the population density. In many cases, the behavior of solutions of such reaction-diffusion equations is determined by the nature of the equilibrium states. Those in turn can often be described via such methods as bifurcation theory and linealized stability analysis, which immediately lead to problems in linear spectral theory. Both the modeling and the analysis can introduce considerations which require the study of elliptic eigenvalue problems with indefinite weight functions.

Now let $\Omega$ be a bounded domain of Euclidean space $\mathbf{R}^{n}, n \geq 3$, with boundary $\partial \Omega$ of class $C^{2+\theta}$ with exponent $0<\theta<1$; its closure $\bar{\Omega}=\Omega \cup \partial \Omega$ is an $n$ dimensional, compact manifold with boundary. The dynamics of a population inhabiting a strongly heterogeneous environment are modeled by diffusive logistic equations of the form

$$
\begin{cases}\frac{\partial w}{\partial t}=d \Delta w+(m(x)-h(x) w) w & \text { in } \Omega \times(0, \infty) \\ w=0 & \text { on } \partial \Omega \times(0, \infty) \\ \left.w\right|_{t=0}=u_{0} & \text { in } \Omega\end{cases}
$$

Here:

(1) $\Delta=\partial^{2} / \partial x_{1}^{2}+\partial^{2} / \partial x_{2}^{2}+\cdots+\partial^{2} / \partial x_{n}^{2}$ is the usual Laplacian.

(2) $d$ is a positive parameter.

(3) $m(x)$ is a real-valued function on $\bar{\Omega}$.

(4) $h(x)$ is a nonnegative function on $\bar{\Omega}$.

This paper is devoted to the study of the existence of positive solutions of problem (1.1), and is an expanded and revised version of the previous paper Taira $[26]$.

First, we discuss our motivation and some of the modeling process leading to problem (1.1). The basic interpretation of the various terms in problem (1.1) is 
that the solution $w\left(x, t ; u_{0}\right)$ represents the population density of a species inhabiting a region $\Omega$. The members of the population are assumed to move about $\Omega$ via the type of random walks occurring in Brownian motion which is modeled by the diffusive term $d \Delta$; hence $d$ represents the rate of diffusive dispersal, so large values of $d$ the population spreads more rapidly than for small values of $d$. The local rate of change in the population density is described by the density dependent term $m(x)-h(x) u$. In this term, $m(x)$ describes the rate at which the population would grow or decline at the location $x$ in the absence of crowding or limitations on the availability of resources. The sign of $m(x)$ will be positive on favorable habitats for population growth and negative on unfavorable ones. Specifically $m(x)$ may be considered as a food source or any resource that will be good in some areas and bad in others. The term $-h(x) u$ describes the effects of crowding on the growth rate of the population at the location $x$; these effects are assumed to be independent of those determining the growth rate. The size of $h(x)$ describes the strength of the effects of crowding within the population.

On the other hand, in terms of biology, the homogeneous Dirichlet condition represents that $\Omega$ is surrounded by a completely hostile exterior such that any member of the population which reaches the boundary dies immediately; in other words, the exterior of the domain is deadly to the population. If the exterior is hostile but not completely deadly, a mixed or Robin boundary condition results, and the analysis is similar.

The basic ecological content of our results is that, for a species with a given rate of diffusion the worst environments are those where favorable and unfavorable regions are closely intermingled, producing "cancellation" effects, and the best are those where the favorable regions are relatively large and few in numbers. This conclusion has significant implications for the design of wildlife refuges. It suggests that a small number of large preserves will provide better protection for a species modeled by problem (1.1) than many small ones, and if the preserves are too small and too closely intermingled with regions where the environment has been damaged, they may not effectively protect the species from extinction.

To study problem (1.1), we may view it as generating a dynamical system. The semilinear parabolic initial boundary value problem (1.1) admits a unique classical solution for sufficiently small times. However, comparison theorems based on the maximum principle guarantee the existence of global solutions in time, since the nonlinearity we are dealing with is sublinear. We show that problem (1.1) admits a unique positive steady state which is a global attractor for nonnegative solutions provided $d$ is sufficiently small, so that the population persists, and further we show that the zero solution is a global attractor for nonnegative solutions if $d$ is sufficiently large, so that the population tends to extinction.

Our models are shown to possess a unique positive steady state, that is, a unique positive solution of the problem

$$
\begin{cases}d \Delta u+(m(x)-h(x) u) u=0 & \text { in } \Omega, \\ u=0 & \text { on } \partial \Omega .\end{cases}
$$


A solution $u \in C^{2}(\bar{\Omega})$ of problem (1.2) is said to be nontrivial if it does not identically equal zero on $\bar{\Omega}$. A nontrivial solution $u$ is called a positive solution if it is strictly positive everywhere in $\Omega$. The object of the analysis is to determine how the spatial arrangement of favorable and unfavorable habitats affects the population being modeled. As is frequently the case, we find that many of the qualitative aspects of the analysis depend crucially on the size of the first positive eigenvalue $\lambda_{1}(m)$ for the linearized Dirichlet problem with indefinite weight function $m(x)$ and positive parameter $\lambda=1 / d$ :

$$
\begin{cases}-\Delta \phi=\lambda m(x) \phi & \text { in } \Omega \\ \phi=0 & \text { on } \partial \Omega .\end{cases}
$$

The next theorem asserts the existence of the first positive eigenvalue $\lambda_{1}(m)$ of problem (1.3), implying persistence for the population (see Manes-Micheletti [16], de Figueiredo [7]):

Theorem 1.1. If $m(x)$ is a function in $L^{\infty}(\Omega)$ such that the set $\{x \in \Omega$ : $m(x)>0\}$ has positive measure, then the first eigenvalue $\lambda_{1}(m)$ of problem (1.3) is positive and simple, and its associated eigenfunction $\phi_{1}(x)$ may be chosen to be strictly positive everywhere in $\Omega$. Moreover, no other eigenvalues have positive eigenfunctions.

By the celebrated Rayleigh theorem (see Manes-Micheletti [16], de Figueiredo [7]), we know that the first eigenvalue $\lambda_{1}(m)$ is given by the variational formula

$$
\lambda_{1}(m)=\inf \left\{\frac{\int_{\Omega}|\nabla \phi|^{2} d x}{\int_{\Omega} m(x) \phi^{2} d x}: \phi \in W_{0}^{1,2}(\Omega), \int_{\Omega} m(x) \phi^{2} d x>0\right\} .
$$

Here $W_{0}^{1,2}(\Omega)$ is the closure of smooth functions with compact support in $\Omega$ in the Sobolev space $W^{1,2}(\Omega)$. By formula (1.4), we find that $\lambda_{1}(m)$ is strictly decreasing with respect to $m(x)$ in the sense that if $m_{1}(x) \leq m_{2}(x)$ almost everywhere in $\Omega$, then the corresponding first eigenvalues $\lambda_{1}\left(m_{1}\right)$ and $\lambda_{1}\left(m_{2}\right)$ satisfy the relation

$$
\lambda_{1}\left(m_{1}\right) \geq \lambda_{1}\left(m_{2}\right)
$$

If the inequality is strict on a set of positive measure, then it follows that $\lambda_{1}\left(m_{1}\right)>$ $\lambda_{1}\left(m_{2}\right)$.

A biological interpretation of Theorem 1.1 is that if there is a favorable region, then the models we consider predict persistence for a population, since the existence of the first positive eigenvalue is equivalent to the existence of a positive density function describing the distribution of the population of $\Omega$. The size of $\lambda_{1}(m)$ is of crucial importance; increasing $\lambda_{1}(m)$ imposes a more stringent condition on the diffusion rate $d$ if the population is to persist, since $0<d<1 / \lambda_{1}(m)$ 
(see Theorem 1.2 or Figure 1.2). It is worthwhile to point out here that the first eigenvalue $\lambda_{1}(m)$ will tend to be smaller in situations where favorable and unfavorable habitats are closely intermingled (producing cancellation effects), and larger when the favorable region consists of a relatively small number of relatively large isolated components.

First, we study problem (1.2) with $d=1 / \lambda$ :

$$
\begin{cases}-\Delta u=\lambda(m(x)-h(x) u) u & \text { in } \Omega, \\ u=0 & \text { on } \partial \Omega .\end{cases}
$$

We assume that $h(x)$ is a nonnegative function in $C^{1}(\bar{\Omega})$, and let

$$
\Omega^{+}(h)=\{x \in \Omega: h(x)>0\}
$$

and

$$
\Omega_{0}(h)=\Omega \backslash \overline{\Omega^{+}(h)} .
$$

Our fundamental hypothesis on the function $h(x)$ is the following (see Figure $1.1)$ :

(Z) The open set $\Omega_{0}(h)$ consists of a finite number of connected components with boundary of class $C^{2+\theta}$, say $\Omega_{0}^{i}(h), 1 \leq i \leq N$, which are bounded away from $\partial \Omega$.

We consider the Dirichlet eigenvalue problem with indefinite weight function $m(x)$ in each connected component $\Omega_{0}^{i}(h)$

$$
\begin{cases}-\Delta \psi=\mu m(x) \psi & \text { in } \Omega_{0}^{i}(h), \\ \psi=0 & \text { on } \partial \Omega_{0}^{i}(h),\end{cases}
$$

and let

$$
\mu_{1}\left(\Omega_{0}^{i}(h)\right)=\text { the first eigenvalue of problem }(1.7)
$$

Recall that the first eigenvalue $\mu_{1}\left(\Omega_{0}^{i}(h)\right)$ is given by the variational formula

$$
\begin{aligned}
& \mu_{1}\left(\Omega_{0}^{i}(h)\right) \\
& =\inf \left\{\frac{\int_{\Omega_{0}^{i}(h)}|\nabla \psi|^{2} d x}{\int_{\Omega_{0}^{i}(h)} m(x) \psi^{2} d x}: \psi \in W_{0}^{1,2}\left(\Omega_{0}^{i}(h)\right), \int_{\Omega_{0}^{i}(h)} m(x) \psi^{2} d x>0\right\} .
\end{aligned}
$$




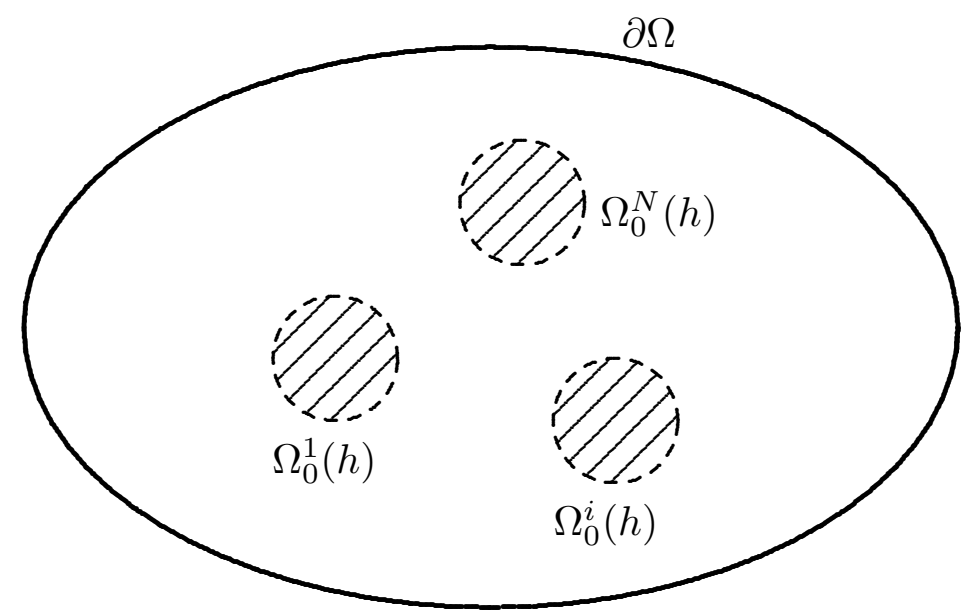

Figure 1.1

If we let

$$
\mu_{1}\left(\Omega_{0}(h)\right)=\min \left\{\mu_{1}\left(\Omega_{0}^{1}(h)\right), \mu_{1}\left(\Omega_{0}^{2}(h)\right), \ldots, \mu_{1}\left(\Omega_{0}^{N}(h)\right)\right\}
$$

then we can state our main result that is a generalization of Cantrell-Cosner [5, Theorems 2.1 and 2.3], Hess-Kato [13, Theorem 2] and Hess [12, Theorem 27.1] to the case where $h(x)$ may vanish in $\Omega$ :

Theorem 1.2. Assume that $h(x) \in C^{1}(\bar{\Omega})$ satisfies condition (Z). If $m(x)$ is a function in $C^{\theta}(\bar{\Omega})$ such that each set $\left\{x \in \Omega_{0}^{i}(h): m(x)>0\right\}, 1 \leq i \leq N$, has positive measure, then problem (1.6) has a unique positive solution $u(\lambda) \in C^{2+\theta}(\bar{\Omega})$ for every $\lambda \in\left(\lambda_{1}(m), \mu_{1}\left(\Omega_{0}(h)\right)\right)$. For any $\lambda \geq \mu_{1}\left(\Omega_{0}(h)\right)$, there exists no positive solution of problem (1.6). Moreover, we have

$$
\lim _{\lambda \rightarrow \mu_{1}\left(\Omega_{0}(h)\right)}\|u(\lambda)\|_{L^{2}(\Omega)}=+\infty
$$

and also

$$
\lim _{\lambda \rightarrow \lambda_{1}(m)}\|u(\lambda)\|_{C^{2+\theta}(\bar{\Omega})}=0
$$

A biological interpretation of Theorem 1.2 is that if the environment has a completely hostile boundary, then the models we consider predict persistence for a population if its diffusion rate $d=1 / \lambda$ is below the critical value $1 / \lambda_{1}(m)$ 
depending on the coefficient $m(x)$ describing the growth rate and if it is above the critical value $1 / \mu_{1}\left(\Omega_{0}(h)\right)$ depending on the coefficient $h(x)$ describing the strength of the crowding effects. Theorem 1.2 also asserts that, in a certain sense, the most favorable situations will occur if there is a relatively large favorable region (with good resources and without crowding effects) located some distance away from the boundary of $\Omega$. The situation may be represented schematically by Figure 1.2.

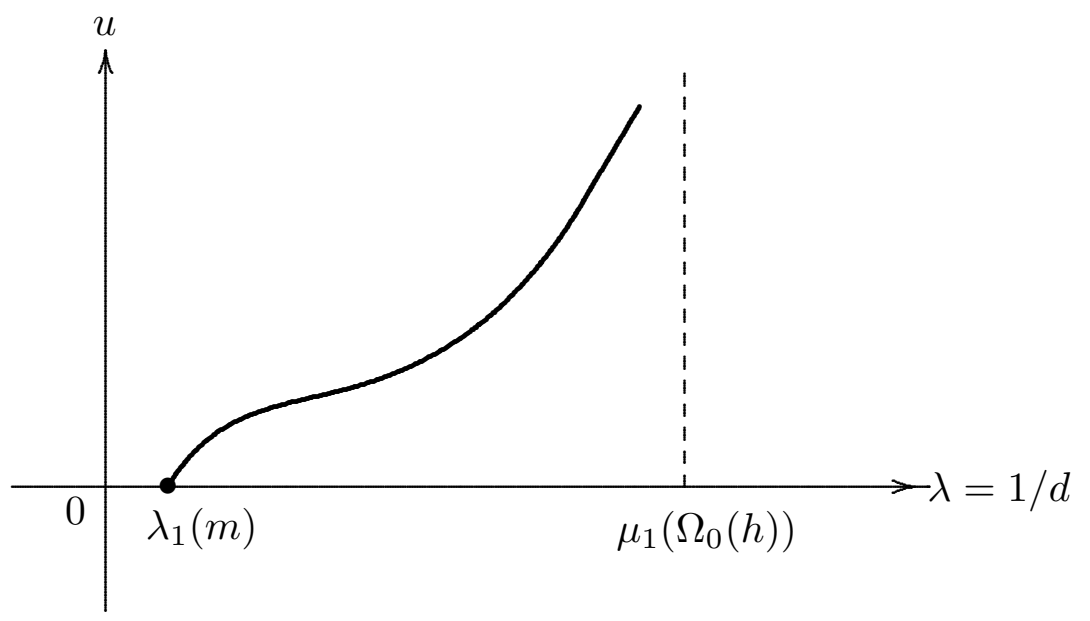

Figure 1.2

Some important remarks are in order.

Remark 1.1. Theorem 1.2 may be proved by using the super-sub-solution method just as in the proof of Fraile et al. [9, Theorems 3.5 and 4.6], if assertion (1.8) is replaced by a weaker one

$$
\lim _{\lambda \rightarrow \mu_{1}\left(\Omega_{0}(h)\right)}\|u(\lambda)\|_{C(\bar{\Omega})}=+\infty .
$$

Theorem 1.2 asserts that assertion (1.8) holds true if the dimension $n$ is greater than $2(n \geq 3)$. It should be emphasized that an estimate of the growth rate of the total size $\|u(\lambda)\|_{L^{1}(\Omega)}=\int_{\Omega} u(\lambda) d x$ of the positive steady states $u(\lambda)$ as $\lambda \uparrow \mu_{1}\left(\Omega_{0}(h)\right)$ is of crucial importance from the viewpoint of population dynamics.

Remark 1.2. López-Gómez and Sabina de Lis [15] analyze the pointwise growth to infinity of positive solutions of the logistic Dirichlet problem in the case where $m(x) \equiv 1$ in $\Omega$ (see [15, Theorems 4.2 and 4.3]). Furthermore, García-Melián et al. [10] study the pointwise behavior and the uniqueness of positive solutions of nonlinear elliptic boundary value problems of general sublinear type, and give the 
exact limiting profile of the positive solutions (see [10, Theorem 3.1, Corollary 3.3 and Theorem 6.4]). Their numerical computations confirm and illuminate the above bifurcation diagram 1.2.

Remark 1.3. Assume that $h(x)>0$ on $\bar{\Omega}$, and that the function $m(x)$ attains positive values in $\Omega$. Then, arguing as in the proof of Cantrell-Cosner [5, Theorem 4.1] we can replace assertion (1.9) by an estimate of the decay rate of the total size of the positive steady states $u(\lambda)$ as $\lambda \downarrow \lambda_{1}(m)$ :

$$
\int_{\Omega} u(\lambda) d x \leq\left(1-\frac{\lambda_{1}(m)}{\lambda}\right)|\Omega|^{2 / 3} \frac{\left(\int_{\Omega}\left(m^{+}\right)^{3} d x\right)^{1 / 3}}{\min _{x \in \bar{\Omega}} h(x)}, \quad \lambda>\lambda_{1}(m) .
$$

Here $|\Omega|$ is the volume of $\Omega$ and

$$
m^{+}(x)=\max \{m(x), 0\}, \quad x \in \Omega \text {. }
$$

Secondly, we study the asymptotic stability properties for positive solutions of problem (1.6). To do this, we consider the semilinear initial boundary value problem (1.1) with $d=1 / \lambda$ :

$$
\begin{cases}\frac{\partial w}{\partial t}=\frac{1}{\lambda} \Delta w+(m(x)-h(x) w) w & \text { in } \Omega \times(0, \infty), \\ w=0 & \text { on } \partial \Omega \times(0, \infty), \\ \left.w\right|_{t=0}=u_{0} & \text { in } \Omega .\end{cases}
$$

It is known (see Amann [3, Theorem 4.5]) that problem (1.11) admits a unique classical global solution $w\left(x, t ; u_{0}\right)$ for each initial value $u_{0} \in C^{2+\theta}(\bar{\Omega})$ satisfying the conditions

$$
\begin{cases}u_{0} \geq 0 & \text { in } \Omega, \\ u_{0}=0 & \text { on } \partial \Omega .\end{cases}
$$

A positive solution $w_{0}(x)$ of problem (1.6) is said to be globally asymptotically stable if we have

$$
\max _{x \in \bar{\Omega}}\left|w\left(x, t ; u_{0}\right)-w_{0}(x)\right| \longrightarrow 0 \quad \text { as } t \rightarrow \infty
$$

for each nontrivial $u_{0} \in C^{2+\theta}(\bar{\Omega})$ satisfying conditions (1.12).

The next theorem describes the asymptotic stability properties for positive solutions of problem (1.6) (cf. Cantrell-Cosner [5, Theorems 2.1 and 4.9], Fraile et al. [9, Theorem 3.7]):

Theorem 1.3. (i) The zero solution of problem (1.6) is globally asymptotically stable if $\lambda$ is so small that

$$
0<\lambda<\lambda_{1}(m)
$$


In this case we can give an estimate of the decay rate of the total size of the population as $t \downarrow 0$ :

$$
\begin{aligned}
& \int_{\Omega} w\left(x, t ; u_{0}\right) d x \\
\leq & \exp \left[-\left(\frac{1}{\lambda}-\frac{1}{\lambda_{1}(m)}\right) \lambda_{1}(1) t\right]|\Omega|^{1 / 2}\left(\int_{\Omega} u_{0}(x)^{2} d x\right)^{1 / 2}, \quad t>0 .
\end{aligned}
$$

(ii) A positive solution $u(\lambda)$ of problem (1.6) is globally asymptotically stable for each $\lambda$ satisfying the condition

$$
\lambda_{1}(m)<\lambda<\mu_{1}\left(\Omega_{0}(h)\right) .
$$

(iii) If $\lambda$ is so large that

$$
\lambda>\mu_{1}\left(\Omega_{0}(h)\right),
$$

then we have

$$
\max _{x \in \bar{\Omega}}\left|w\left(x, t ; u_{0}\right)\right| \longrightarrow \infty \quad \text { as } t \rightarrow \infty
$$

for each nontrivial $u_{0} \in C^{2+\theta}(\bar{\Omega})$ satisfying conditions (1.12).

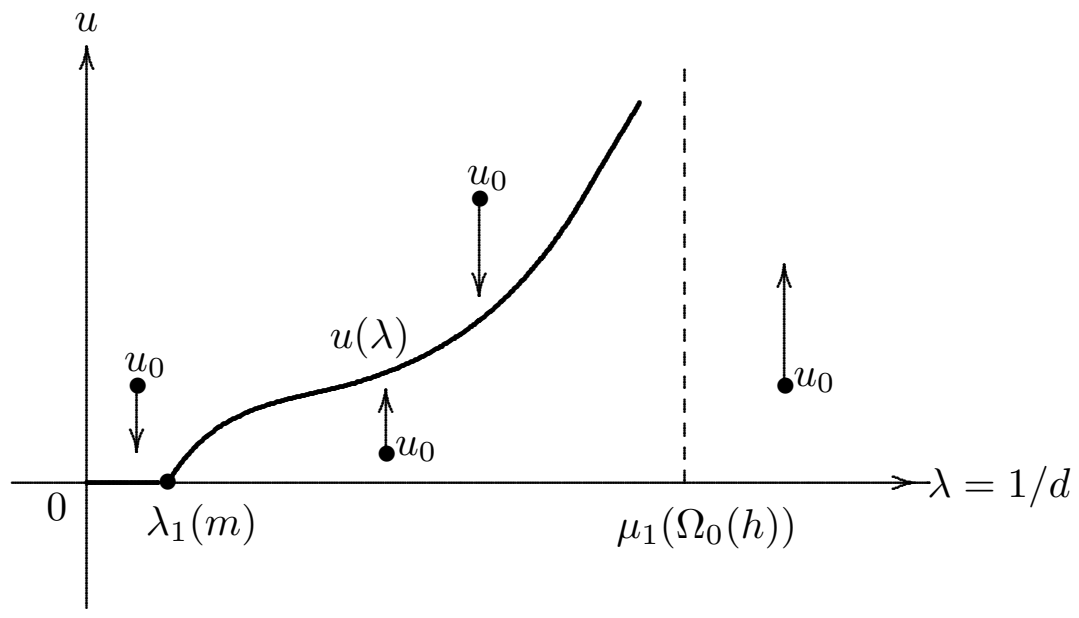

Figure 1.3

A biological interpretation of Theorem 1.3 is that a population will grow exponentially until limited by lack of available resources if the diffusion rate $d=1 / \lambda$ is below the critical value $1 / \mu_{1}\left(\Omega_{0}(h)\right)$; this idea is generally credited to Thomas Malthus. On the other hand, if the diffusion rate $d=1 / \lambda$ is above the critical value $1 / \mu_{1}\left(\Omega_{0}(h)\right)$, then the model obeys the logistic equation introduced by P. F. 
Verhulst around 1840. The situation may be represented schematically by Figure 1.3 .

The rest of this paper is organized as follows. First, in Section 2, by using Green's formula we prove that if there exists a positive solution $u(\lambda) \in C^{2}(\bar{\Omega})$ of problem (1.6), then we have $\lambda>\lambda_{1}(m)$ (Lemma 2.1). Next, by making use of the implicit function theorem we prove that there exists a critical value $\lambda^{*} \in$ $\left(\lambda_{1}(m),+\infty\right]$ such that problem (1.6) has a positive solution $u(\lambda)$ for all $\lambda \in$ $\left(\lambda_{1}(m), \lambda^{*}\right)$ (Lemma 2.5). Moreover, we prove the inequality (Lemma 2.7)

$$
\lambda^{*} \leq \mu_{1}\left(\Omega_{0}(h)\right) .
$$

The proof of formula $\lambda^{*}=\mu_{1}\left(\Omega_{0}(h)\right)$, that is, the reverse inequality of inequality (1.14)

$$
\mu_{1}\left(\Omega_{0}(h)\right) \leq \lambda^{*}
$$

is carried out in Section 3 through Section 5, just as in Taira [25] and also Ouyang [17].

In Section 3 we study the behavior of the positive solutions $u(\lambda), 0<\lambda<\lambda^{*}$, in the set $\Omega^{+}(h)$. Roughly speaking, we prove that, for each $\varepsilon>0$ there exists a constant $C(\varepsilon, \lambda)>0$ such that (Lemma 3.2)

$$
(u(\lambda)(x))^{p-1-\varepsilon} \leq \frac{C(\varepsilon, \lambda)}{h(x)} \text { for all } x \in \Omega^{+}(h)
$$

This is an essential step in the proof of inequality (1.15) in Section 5 (see estimate (5.8)). On the other hand, in Section 4, we prove that (Lemma 4.1)

$$
\lim _{\lambda \rightarrow \lambda^{*}}\|u(\lambda)\|_{L^{2}(\Omega)}=+\infty .
$$

Namely we show that the solution $u(\lambda)$ blows up at the critical value $\lambda^{*}$. In Section 5 , we prove that the critical value $\lambda^{*}$ is an eigenvalue of the Dirichlet problem (1.7) (Proposition 5.1), which implies the desired reverse inequality (1.15).

In Section 6 we prove Theorem 1.3 by using comparison theorems based on the maximum principle just as in Fraile et al. [9, Theorem 3.7], Pao [18, Chapter 5, Theorem 4.4] and Sattinger [22, Theorem 2.6.2].

If the boundary acts as a barrier, so that individuals reaching the boundary simply return to the interior, a Neumann boundary condition results. The analysis may be somewhat different, since the operator $-\Delta$ will have zero as an eigenvalue. However, the same general approach can still be used. In the final Section 7 we study the problem with homogeneous Neumann condition

$$
\begin{cases}\frac{\partial w}{\partial t}=d \Delta w+(m(x)-h(x) w) w & \text { in } \Omega \times(0, \infty), \\ \frac{\partial w}{\partial \mathbf{n}}=0 & \text { on } \partial \Omega \times(0, \infty), \\ w=u_{0} & \text { in } \Omega\end{cases}
$$


where $\mathbf{n}$ is the unit exterior normal to $\partial \Omega$.

In the context of population dynamics, the behavior of solutions of problem (1.16) is similar to that of problem (1.1) with homogeneous Dirichlet condition if $\int_{\Omega} m(x) d x<0$ (Theorem 7.3); so that there is a positive eigenvalue with positive eigenfunction to act as a bifurcation point for positive steady states (Theorem 7.1). If $\int_{\Omega} m(x) d x \geq 0$, then there will exist positive steady states for all values of $d$ (Theorem 7.2). A biological interpretation is that when the environment has an impassable boundary and is on the average unfavorable $\left(\int_{\Omega} m(x) d x<0\right)$, then high diffusion rates have the same effect (that is, the ultimate extinction of the population) as they always have when the boundary is deadly; but if the boundary is impassable and the environment is on the average neutral or favorable $\left(\int_{\Omega} m(x) d x \geq 0\right)$, then the population can persist, no matter what its rate of diffusion (see Hess [12, Example 28.6]).

In Appendix we prove the decay estimate (1.10) in Remark 1.3.

The author would like to thank Professor Masayasu Mimura for his helpful suggestions on the formulation of Theorem 1.3 from the point of view of mathematical ecology. This research is partially supported by Grant-in-Aid for General Scientific Research (No. 13440041), Ministry of Education, Culture, Sports, Science and Technology, Japan.

\section{Proof of Theorem 1.2 -(1)-}

Step I: First, we begin with the following lower bound on the parameter $\lambda$ for the existence of positive solutions of problem (1.6):

Lemma 2.1. Assume that $h(x) \geq 0$ in $\Omega$. If there exists a positive solution $u \in C^{2}(\bar{\Omega})$ of problem (1.6), then we have

$$
\lambda>\lambda_{1}(m) .
$$

Proof. Let $u \in C^{2}(\bar{\Omega})$ be a positive solution of problem (1.6)

$$
\begin{cases}-\Delta u=\lambda(m(x)-h(x) u) u & \text { in } \Omega, \\ u>0 & \text { in } \Omega, \\ u=0 & \text { on } \partial \Omega .\end{cases}
$$

Then, applying Theorem 1.1 to our situation we obtain that

$$
\lambda=\lambda_{1}(m(x)-h(x) u) .
$$


However, since $h(x) \geq 0$ in $\Omega$, it follows that

$$
m(x)-h(x) u \leq m(x) \quad \text { in } \Omega
$$

By assertion (1.5), we have

$$
\lambda_{1}(m(x)-h(x) u)>\lambda_{1}(m(x))
$$

Therefore, the desired lower bound (2.1) follows by combining assertions (2.2) and (2.3).

Step II: Next we prove that there exists a critical value $\lambda^{*} \in\left(\lambda_{1}(m),+\infty\right]$ such that the solution curve $(\lambda, u(\lambda))$ may be parametrized by $\lambda, \lambda_{1}(m)<\lambda<\lambda^{*}$, as a curve of class $C^{1}$.

To do this, we let

$$
C_{0}^{2+\theta}(\bar{\Omega})=\left\{u \in C^{2+\theta}(\bar{\Omega}): u=0 \text { on } \partial \Omega\right\}
$$

and associate with problem (1.6) a nonlinear mapping $F(\lambda, u)$ of $\mathbf{R} \times C_{0}^{2+\theta}(\bar{\Omega})$ into $C^{\theta}(\bar{\Omega})$ as follows:

$$
\begin{aligned}
F: \mathbf{R} \times C_{0}^{2+\theta}(\bar{\Omega}) & \longrightarrow C^{\theta}(\bar{\Omega}) \\
(\lambda, u) & \longmapsto-\Delta u-\lambda m(x) u+\lambda h(x) u^{2} .
\end{aligned}
$$

It is clear that a function $u \in C^{2+\theta}(\bar{\Omega})$ is a solution of problem (1.6) if and only if $F(\lambda, u)=0$.

Step II-a: First, the next lemma proves the existence of positive solutions of problem (1.6) bifurcating at $\left(\lambda_{1}(m), 0\right)$ :

Lemma 2.2. There exists an unbounded continuum $\mathcal{C}$ of positive solutions of problem (1.6) emanating from $\left(\lambda_{1}(m), 0\right)$.

Proof. (1) We shall transpose the nonlinear problem (1.6) into an operator equation for the resolvent $K$ of the Dirichlet problem in an appropriate ordered Banach space, just as in Amann [2] and Hess-Kato [13].

We let

$$
E:=C_{0}(\bar{\Omega})=\{v \in C(\bar{\Omega}): v=0 \text { on } \partial \Omega\} .
$$

This is an ordered Banach space with the natural ordering given by the positive cone

$$
P_{E}=\left\{v \in C_{0}(\bar{\Omega}): v \geq 0 \text { in } \Omega\right\} .
$$


We shall primarily work in the naturally ordered Banach space

$$
X:=C_{0}^{1}(\bar{\Omega})=\left\{v \in C^{1}(\bar{\Omega}): v=0 \text { on } \partial \Omega\right\},
$$

with positive cone

$$
P_{X}=\left\{v \in C_{0}^{1}(\bar{\Omega}): v \geq 0 \text { in } \Omega\right\} .
$$

It is easy to verify that the interior Int $\left(P_{X}\right)$ of $P_{X}$ is characterized as follows:

$$
\operatorname{Int}\left(P_{X}\right)=\left\{v \in C_{0}^{1}(\bar{\Omega}): v>0 \text { in } \Omega, \frac{\partial v}{\partial \mathbf{n}}<0 \text { on } \partial \Omega\right\}
$$

where $\mathbf{n}$ is the unit exterior normal to $\partial \Omega$.

If $1<p<\infty$, we can introduce a continuous linear operator

$$
K: L^{p}(\Omega) \longrightarrow W^{2, p}(\Omega) \cap W_{0}^{1, p}(\Omega)
$$

as follows (see Gilbarg-Trudinger [11]): For any $g \in L^{p}(\Omega)$, the function $u=K g \in$ $W^{2, p}(\Omega) \cap W_{0}^{1, p}(\Omega)$ is the unique solution of the Dirichlet problem

$$
\begin{cases}-\Delta u=g & \text { in } \Omega, \\ u=0 & \text { on } \partial \Omega .\end{cases}
$$

Then, by the Ascoli-Arzelà theorem we find that the operator $K$, considered as

$$
K: C(\bar{\Omega}) \longrightarrow C_{0}^{1}(\bar{\Omega})
$$

is compact. Indeed, it follows from an application of Sobolev's imbedding theorem that $W^{2, p}(\Omega)$ is continuously imbedded into $C^{2-n / p}(\bar{\Omega})$ for all $n<p<\infty$. Moreover, by the strong maximum principle it follows that $K$ is strongly positive, that is, $K g \in \operatorname{Int}\left(P_{X}\right)$ for all $g \in P_{E} \backslash\{0\}$.

Finally, it is easy to verify that a function $u$ is a solution of problem (1.6) if and only if it satisfies the equation

$$
u=\lambda K\left(m(x) u-h(x) u^{2}\right) \quad \text { in } C_{0}^{1}(\bar{\Omega}) .
$$

Indeed, it suffices to note that $m(x) \in C^{\theta}(\bar{\Omega}), h(x) \in C^{1}(\bar{\Omega})$ and that $K$ maps $C^{\theta}(\bar{\Omega})$ continuously into $C_{0}^{2+\theta}(\bar{\Omega})$.

(2) Just as in the proof of Hess-Kato [13, Theorem 2], we extend the function

$$
f(x, s)=m(x) s-h(x) s^{2}
$$

as an odd function in the variable $s$ as follows:

$$
\tilde{f}(x, s)= \begin{cases}m(x) s-h(x) s^{2} & \text { if } s>0 \\ m(x) s+h(x) s^{2} & \text { if } s \leq 0 .\end{cases}
$$


Then we associate with $\tilde{f}(x, s)$ the Nemytskii operator $\tilde{F}(u)$ defined by the formula

$$
\tilde{F}(u)=\tilde{f}(x, u(x)), \quad x \in \bar{\Omega},
$$

and consider instead of equation (2.4) the following:

$$
u=\lambda K \tilde{F}(u) \quad \text { in } C_{0}^{1}(\bar{\Omega}) .
$$

We remark that $u$ is a solution of equation (2.5) if and only if $-u$ is a solution; hence we may identify positive solutions with negative solutions in what follows.

(3) Now the Crandall-Rabinowitz local bifurcation theorem [6, Theorem 1.7] may be employed to assert that the simplicity of $\lambda_{1}(m)$ guarantees the existence of the continuum of nontrivial solutions of problem (1.6) emanating from $\left(\lambda_{1}(m), 0\right)$, which can be expressed as the union of two subcontinua intersecting at $\left(\lambda_{1}(m), 0\right)$ (cf. Deimling [8, Corollary 29.1]).

However, by the compactness and strong positivity of $K$ it follows that these subcontinua are locally the strictly positive and the strictly negative solutions of equation (2.5).

Indeed, assume, to the contrary, that there exists a sequence $\left(\lambda_{j}, u_{j}\right)$ with $\lambda_{j}>$ $0, u_{j} \in X$, such that

$$
\begin{aligned}
& u_{j}=\lambda_{j} K \tilde{F}\left(u_{j}\right), \\
& \lambda_{j} \rightarrow \lambda_{1}(m), \\
& u_{j} \rightarrow 0 \quad \text { in } X, \\
& u_{j} \notin \operatorname{Int}\left(P_{X}\right) .
\end{aligned}
$$

If we let

$$
v_{j}=\frac{u_{j}}{\left\|u_{j}\right\|_{X}}
$$

then it follows that

$$
\begin{aligned}
& v_{j} \notin \operatorname{Int}\left(P_{X}\right) \\
& \left\|v_{j}\right\|_{X}=1 \\
& v_{j}=\lambda_{j} \frac{K \tilde{F}\left(u_{j}\right)}{\left\|u_{j}\right\|_{X}} .
\end{aligned}
$$

By the compactness of $K$, we may choose a subsequence, denoted again by $\left\{v_{j}\right\}$, which converges to some function $v$ in $X$. Therefore, passing to the limit we obtain that

$$
\begin{aligned}
& v \notin \operatorname{Int}\left(P_{X}\right), \\
& \|v\|_{X}=1,
\end{aligned}
$$


and that

$$
v=\lambda_{1}(m) K(m(x) v)
$$

or equivalently,

$$
-\Delta v=\lambda_{1}(m) m(x) v .
$$

However, we arrive at a contradiction

$$
v \in \operatorname{Int}\left(P_{X}\right),
$$

since $\lambda_{1}(m)$ is a simple eigenvalue of problem (1.3) having a positive eigenfunction in $\operatorname{Int}\left(P_{X}\right)$.

(4) We show that these subcontinua are globally the strictly positive and the strictly negative solutions of equation (2.5) as in Figure 2.1.

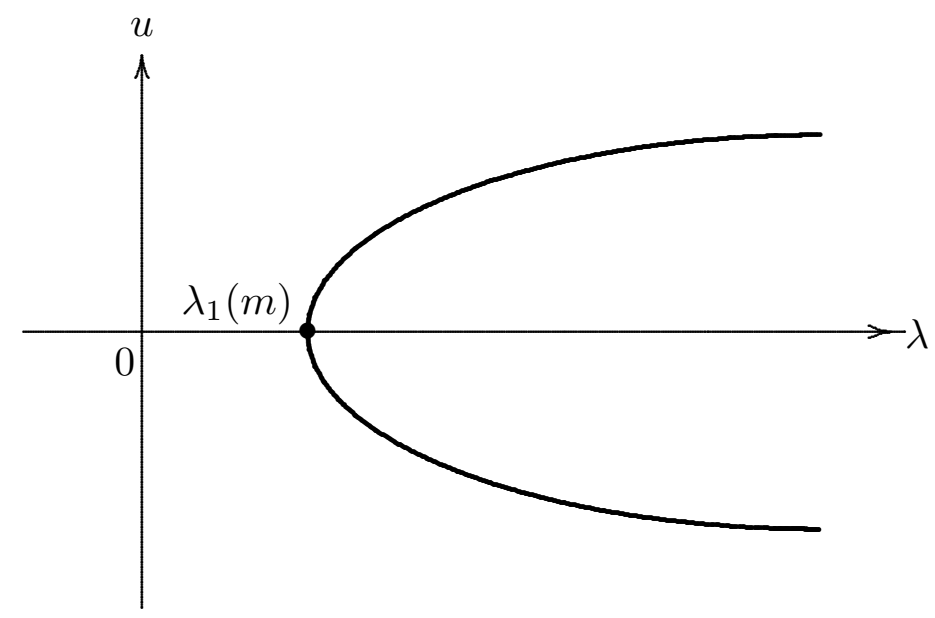

Figure 2.1

Assume, to the contrary, that there exists a point $\left(\lambda_{0}, u_{0}\right)$ such that $\lambda_{0}>0$ and $u_{0} \in \partial P_{X}$ with $u_{0}>0$. We let

$$
c=\max _{\substack{x \in \bar{\Omega} \\ 0 \leq s \leq\left\|u_{0}\right\|}}|m(x)-h(x) s|+1 .
$$

Then it follows that

$$
\begin{aligned}
\left(-\Delta+\lambda_{0} c\right) u_{0} & \left.=\lambda_{0}\left(F\left(u_{0}\right)\right)+c u_{0}\right) \\
& =\lambda_{0} u_{0}\left(m(x)-h(x) u_{0}+c\right)>0 \quad \text { in } \Omega .
\end{aligned}
$$

Hence, by the maximum principle we arrive at a contradiction

$$
u_{0} \in \operatorname{Int}\left(P_{X}\right)
$$


On the other hand, it is clear that equation (2.5) has no nontrivial solutions for $\lambda=0$.

(5) Moreover, the Rabinowitz global bifurcation theorem [19, Theorem 1.10] tells us that the subcontinuum $\mathcal{C}$ of positive solutions emanating from $\left(\lambda_{1}(m), 0\right)$ is either unbounded or contains another bifurcation point $\left(\lambda_{0}, 0\right)$ with $\lambda_{0} \neq \lambda_{1}(m)$.

However, just as in step (3) we can prove that the subcontinuum $\mathcal{C}$ can not contain a point $\left(\lambda_{0}, 0\right)$ with $\lambda_{0} \neq \lambda_{1}(m)$; hence $\mathcal{C}$ must be unbounded (cf. Deimling [8, Theorem 29.2]).

The proof of Lemma 2.2 is now complete.

Step II-b: Secondly, we prove a uniqueness result for positive solutions of problem (1.6) which implies that the unbounded continuum $\mathcal{C}$ is actually an arc:

Lemma 2.3. Assume that $h(x) \geq 0$ in $\Omega$. Then problem (1.6) has at most one positive solution $u(\lambda)$ for any $\lambda>\lambda_{1}(m)$.

Proof. (1) Our proof is based on the following spectral theorem for Schrödinger operators (cf. Reed-Simon [21, Chapter XIII]):

Theorem 2.4. Assume that $q(x)$ is a function in $L^{\infty}(\Omega)$. Then the Dirichlet eigenvalue problem

$$
\begin{cases}-\Delta \psi+q(x) \psi=\sigma \psi & \text { in } \Omega, \\ \psi=0 & \text { on } \partial \Omega\end{cases}
$$

has an infinite sequence of eigenvalues

$$
\sigma_{1}<\sigma_{2} \leq \sigma_{3} \leq \ldots
$$

The first eigenvalue $\sigma_{1}=\sigma_{1}(q)$ is simple and is the only eigenvalue admitting a positive eigenfunction. Moreover, it is strictly increasing with respect to $q(x)$ in the sense that if $q_{1}(x) \leq q_{2}(x)$ in $\Omega$, then the corresponding first eigenvalues $\sigma_{1}\left(q_{1}\right)$ and $\sigma_{1}\left(q_{2}\right)$ satisfy the relation

$$
\sigma_{1}\left(q_{1}\right)<\sigma_{1}\left(q_{2}\right)
$$

(2) Let $u_{i}(x), i=1,2$ be two positive solutions of problem (1.6)

$$
\begin{cases}-\Delta u_{i}=\lambda\left(m(x) u_{i}-h(x) u_{i}^{2}\right) & \text { in } \Omega \\ u_{i}>0 & \text { in } \Omega, \\ u_{i}=0 & \text { on } \partial \Omega .\end{cases}
$$

Then it follows that the Dirichlet eigenvalue problem

$$
\begin{cases}-\Delta v+\lambda\left(h(x) u_{1}-m(x)\right) v=\sigma v & \text { in } \Omega, \\ v=0 & \text { on } \partial \Omega\end{cases}
$$


has a positive solution $v=u_{1}$ with $\sigma=0$. Hence, applying Theorem 2.4 to our situation we obtain that

$$
\sigma_{1}\left(\lambda\left(h(x) u_{1}-m(x)\right)\right)=0 .
$$

On the other hand, we find that the Dirichlet eigenvalue problem

$$
\begin{cases}-\Delta w+\lambda\left(h(x)\left(u_{1}+u_{2}\right)-m(x)\right) w=\sigma w & \text { in } \Omega, \\ w=0 & \text { on } \partial \Omega\end{cases}
$$

has a solution $w=u_{1}-u_{2}$ with $\sigma=0$. However, since $h(x) \geq 0$ in $\Omega$, we have, by assertions (2.6) and (2.7),

$$
\sigma_{1}\left(\lambda\left(h(x)\left(u_{1}+u_{2}\right)-m(x)\right)\right)>\sigma_{1}\left(\lambda\left(h(x) u_{1}-m(x)\right)\right)=0 .
$$

This implies that problem (2.8) with $\sigma=0$ can not have any solutions other than $w=0$, so that $u_{1}(x)=u_{2}(x)$ in $\Omega$.

The proof of Lemma 2.3 is complete.

Step II-c: Thirdly, by using the implicit function theorem we show (cf. Hess [12, Theorem 27.1]) that there exists a critical value $\lambda^{*} \in\left(\lambda_{1}(m),+\infty\right]$ such that we can parametrize the bifurcation solution curve $(\lambda, u(\lambda))$ by $\lambda, \lambda_{1}(m)<\lambda<\lambda^{*}$, as a $C^{1}$ curve as in Figure 2.2.

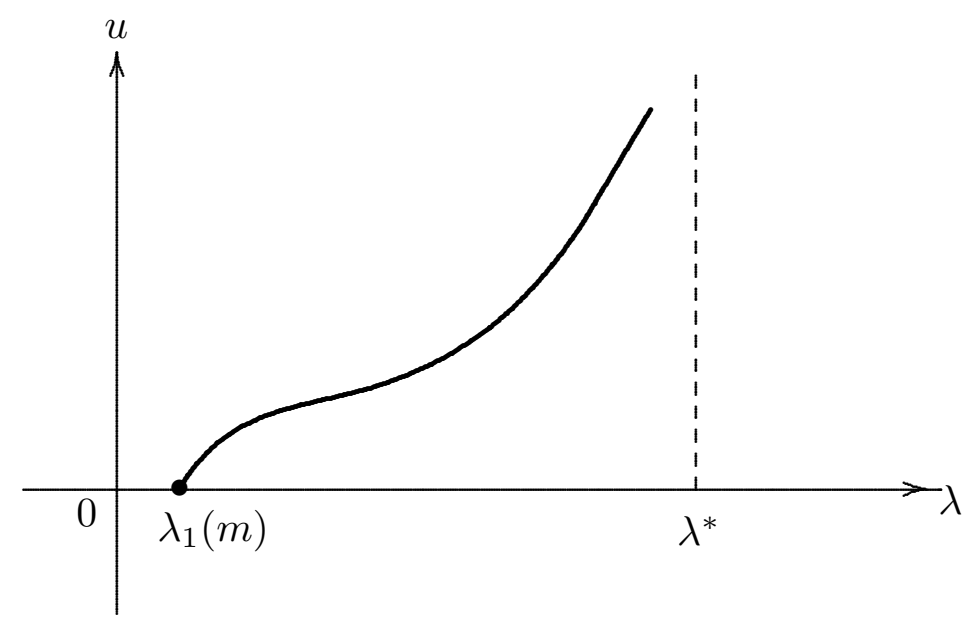

Figure 2.2

Lemma 2.5. There exists a constant $\lambda^{*} \in\left(\lambda_{1}(m),+\infty\right]$ such that we have a positive solution $(\lambda, u(\lambda))$ of the equation $F(\lambda, u)=0$ for all $\lambda \in\left(\lambda_{1}(m), \lambda^{*}\right)$.

Proof. It is known (see Gilbarg-Trudinger [11]) that the Fréchet derivative

$$
F_{u}(\lambda, u(\lambda)): C_{0}^{2+\theta}(\bar{\Omega}) \longrightarrow C^{\theta}(\bar{\Omega})
$$




$$
v \longmapsto-\Delta v-\lambda m(x) v+2 \lambda h(x) u(\lambda) v
$$

is a Fredholm operator with index zero. Hence, to prove the lemma it suffices to show that $F_{u}(\lambda, u(\lambda))$ is injective. Indeed, by using the implicit function theorem we can find a constant $\lambda^{*} \in\left(\lambda_{1}(m),+\infty\right]$ such that $F(\lambda, u(\lambda))=0$ and $F_{u}(\lambda, u(\lambda))$ is an algebraic and topological isomorphism for all $\lambda \in\left(\lambda_{1}(m), \lambda^{*}\right)$.

The next claim proves the injectivity and hence surjectivity of the Fréchet derivative $F_{u}(\lambda, u(\lambda))$ :

Claim 2.6. The first eigenvalue $\mu_{1}(\lambda)$ of $F_{u}(\lambda, u(\lambda))$ is positive for $\lambda>\lambda_{1}(m)$; in particular, 0 is not an eigenvalue of $F_{u}(\lambda, u(\lambda))$.

Proof. Let $u(\lambda)$ be a positive solution of problem (1.6). In view of formula (2.9), we remark that $\mu_{1}(\lambda)$ is the first eigenvalue of the Dirichlet problem

$$
\begin{cases}-\Delta v+(2 \lambda h(x) u(\lambda)-\lambda m(x)) v=\sigma v & \text { in } \Omega \\ v=0 & \text { on } \partial \Omega\end{cases}
$$

so that

$$
\mu_{1}(\lambda)=\sigma_{1}(2 \lambda h(x) u(\lambda)-\lambda m(x))
$$

However, it follows that the Dirichlet eigenvalue problem

$$
\begin{cases}-\Delta v+\lambda(h(x) u(\lambda)-m(x)) v=\sigma v & \text { in } \Omega, \\ v=0 & \text { on } \partial \Omega\end{cases}
$$

has a positive solution $v=u(\lambda)$ with $\sigma=0$. Hence, applying Theorem 2.4 to our situation we obtain that

$$
\sigma_{1}(\lambda(h(x) u(\lambda)-m(x)))=0 .
$$

Therefore, since $h(x) \geq 0$ in $\Omega$, we have, by formulas (2.10) and (2.11),

$$
\mu_{1}(\lambda)=\sigma_{1}(2 \lambda h(x) u(\lambda)-\lambda m(x))>\sigma_{1}(\lambda h(x) u(\lambda)-\lambda m(x))=0 .
$$

This proves Claim 2.6.

The proof of Lemma 2.5 is now complete.

By Lemma 2.5, we have a positive bifurcation solution curve $(\lambda, u(\lambda))$ of the equation $F(\lambda, u)=0$ for all $\lambda \in\left(\lambda_{1}(m), \lambda^{*}\right)$.

Step III: Finally, it remains to characterize the critical value $\lambda^{*}$ as follows:

$$
\lambda^{*}=\mu_{1}\left(\Omega_{0}(h)\right)=\min \left\{\mu_{1}\left(\Omega_{0}^{1}(h)\right), \mu_{1}\left(\Omega_{0}^{2}(h)\right), \ldots, \mu_{1}\left(\Omega_{0}^{N}(h)\right)\right\} .
$$


Step III-a: First, we prove an upper bound on the parameter $\lambda$ for the existence of positive solutions of problem (1.6) which implies that

$$
\lambda^{*} \leq \mu_{1}\left(\Omega_{0}(h)\right)
$$

Lemma 2.7. Assume that $h(x)$ satisfies condition $(Z)$ and that each set $\{x \in$ $\left.\Omega_{0}^{i}(h): m(x)>0\right\}, 1 \leq i \leq l$, has positive measure. If there exists a positive solution $u(\lambda) \in C^{2}(\bar{\Omega})$ of problem (1.6) for $\lambda>0$, then we have

$$
\lambda<\mu_{1}\left(\Omega_{0}(h)\right)=\min \left\{\mu_{1}\left(\Omega_{0}^{1}(h)\right), \mu_{1}\left(\Omega_{0}^{2}(h)\right), \ldots, \mu_{1}\left(\Omega_{0}^{N}(h)\right)\right\} .
$$

Proof. Let $\psi_{1}(x)$ be a positive eigenfunction corresponding to the first eigenvalue $\mu_{1}\left(\Omega_{0}^{i}(h)\right)$ of the Dirichlet problem

$$
\begin{cases}-\Delta \psi=\mu m(x) \psi & \text { in } \Omega_{0}^{i}(h), \\ \psi=0 & \text { on } \partial \Omega_{0}^{i}(h) .\end{cases}
$$

Then it follows that the coercivity condition holds:

$$
\int_{\Omega_{0}^{i}(h)} m(x) \psi_{1}^{2} d x>0 .
$$

Indeed, since the set $\left\{x \in \Omega_{0}^{i}(h): m(x)>0\right\}$ has positive measure, we obtain that $\mu_{1}\left(\Omega_{0}^{i}(h)\right)>0$ and further from formula (1.4) that

$$
\int_{\Omega_{0}^{i}(h)} m(x) \psi_{1}^{2} d x=\frac{\int_{\Omega_{0}^{i}(h)}\left|\nabla \psi_{1}\right|^{2} d x}{\mu_{1}\left(\Omega_{0}^{i}(h)\right)}>0 .
$$

On the other hand, it follows that

$$
-\Delta u(\lambda)=\lambda m(x) u(\lambda)-\lambda h(x) u(\lambda)^{2}=\lambda m(x) u(\lambda) \quad \text { in } \Omega_{0}^{i}(h),
$$

since $h(x)=0$ in $\Omega_{0}^{i}(h)$.

Then we have, by a direct calculation,

$$
\begin{aligned}
\sum_{j=1}^{n} \frac{\partial}{\partial x_{j}}\left(u(\lambda)^{2} \frac{\partial}{\partial x_{j}}\left(\frac{\psi_{1}}{u(\lambda)}\right)\right) & =\Delta \psi_{1} \cdot u(\lambda)-\Delta u(\lambda) \cdot \psi_{1} \\
& =-\mu_{1}\left(\Omega_{0}^{i}(h)\right) m(x) \psi_{1} \cdot u(\lambda)+\lambda m(x) u(\lambda) \cdot \psi_{1} \\
& =\left(\lambda-\mu_{1}\left(\Omega_{0}^{i}(h)\right)\right) m(x) u(\lambda) \cdot \psi_{1} \quad \text { in } \Omega_{0}^{i}(h),
\end{aligned}
$$


and so

$$
\left(\lambda-\mu_{1}\left(\Omega_{0}^{i}(h)\right)\right) m(x) \psi_{1}=\frac{1}{u(\lambda)} \sum_{j=1}^{n} \frac{\partial}{\partial x_{j}}\left(u(\lambda)^{2} \frac{\partial}{\partial x_{j}}\left(\frac{\psi_{1}}{u(\lambda)}\right)\right) \quad \text { in } \Omega_{0}^{i}(h)
$$

Therefore, by integration by parts it follows that

$$
\begin{aligned}
& \left(\lambda-\mu_{1}\left(\Omega_{0}^{i}(h)\right)\right) \int_{\Omega_{0}^{i}(h)} m(x) \psi_{1}^{2} d x \\
= & \int_{\Omega_{0}^{i}(h)} \sum_{j=1}^{n} \frac{\partial}{\partial x_{j}}\left(u(\lambda)^{2} \frac{\partial}{\partial x_{j}}\left(\frac{\psi_{1}}{u(\lambda)}\right)\right) \cdot \frac{\psi_{1}}{u(\lambda)} d x \\
= & -\int_{\Omega_{0}(h)} u(\lambda)^{2} \sum_{j=1}^{n} \frac{\partial}{\partial x_{j}}\left(\frac{\psi_{1}}{u(\lambda)}\right) \frac{\partial}{\partial x_{j}}\left(\frac{\psi_{1}}{u(\lambda)}\right) d x \\
< & 0 .
\end{aligned}
$$

In view of condition (2.15), this proves inequality (2.14).

The proof of Lemma 2.7 is complete.

Step III-b: In Section 5, we shall prove that the critical value $\lambda^{*}$ is an eigenvalue of the Dirichlet problem (1.7) (Proposition 5.1), which implies the desired formula (2.12).

\section{Proof of Theorem $1.2-(2)-$}

In Section 3 through Section 5 we shall prove the reverse inequality of inequality $(2.13)$

$$
\mu_{1}\left(\Omega_{0}(h)\right) \leq \lambda^{*}
$$

First, we begin with the following elementary lemma:

Lemma 3.1. If $h(x)$ is a nonnegative function in $C^{1}(\bar{\Omega})$, then we can construct a function $h^{*}(x) \in C^{1}(\bar{\Omega})$ having the following properties:

(a) $0<h^{*}(x) \leq h(x)$ for all $x \in \Omega_{+}(h)$.

(b) For each $\varepsilon>0$, there exists a constant $C>0$, depending on $\sup _{\Omega}|\nabla h|$, such that

$$
\sup _{\Omega_{+}(h)}\left|\frac{\nabla h^{*}}{\left(h^{*}\right)^{1-\varepsilon}}\right| \leq \frac{C}{\varepsilon^{2}}
$$


Proof. We let

$$
d(x)=\operatorname{dist}\left(x, \partial \Omega_{+}(h)\right), \quad x \in \Omega_{+}(h),
$$

and define a function $h^{*}(x)$ by the formulas

$$
h^{*}(x)= \begin{cases}0 & \text { for } x \in \Omega \backslash \Omega_{+}(h), \\ e^{-\frac{1}{h(x)}} & \text { for } x \in \Omega_{+}(h) \text { and } 0<d(x)<\delta, \\ h(x) & \text { for } x \in \Omega_{+}(h) \text { and } d(x)>2 \delta,\end{cases}
$$

and

$$
0<h^{*}(x) \leq h(x) \quad \text { for } x \in \Omega_{+}(h) \text { and } \delta \leq d(x) \leq 2 \delta .
$$

Then it is easy to verify that the function $h^{*}(x)$ enjoys properties (a) and (b).

For example, property (b) may be verified in the following way. Since we have

$$
\nabla h^{*}(x)=e^{-\frac{1}{h(x)}} \frac{\nabla h(x)}{h(x)^{2}} \quad \text { for } x \in \Omega_{+}(h), 0<d(x)<\delta,
$$

it follows that

$$
\begin{aligned}
\left|\frac{\nabla h^{*}(x)}{h^{*}(x)^{1-\varepsilon}}\right| & =|\nabla h(x)| e^{-\frac{\varepsilon}{h(x)}} \frac{1}{h(x)^{2}} \\
& \leq \sup _{\Omega}|\nabla h| \cdot \sup _{\Omega}\left(\frac{e^{-\frac{\varepsilon}{h}}}{h^{2}}\right) \\
& \leq \sup _{\Omega}|\nabla h|\left(\frac{4 e^{-2}}{\varepsilon^{2}}\right) \cdot \square
\end{aligned}
$$

The next lemma will play an essential role in the proof of inequality (3.1) (see the proof of Proposition 5.1).

Lemma 3.2. If $u(\lambda)$ is a positive solution of problem (1.6) with $\lambda_{1}(m)<\lambda<\lambda^{*}$, then we have, for any $0<\varepsilon<1$,

$$
h^{*} u(\lambda)^{1-\varepsilon} \in L^{\infty}\left(\Omega^{+}(h)\right),
$$

and

$$
\sup _{\Omega^{+}(h)}\left(h^{*} u(\lambda)^{1-\varepsilon}\right) \leq C(\varepsilon, \lambda)
$$

with a constant $C(\varepsilon, \lambda)>0$. Moreover, if $\lambda$ is finite, then so is the constant $C(\varepsilon, \lambda)$. 
Proof. The proof is divided into seven steps just as in the proof of Taira [25, Lemma 4.2].

Without loss of generality, we may assume that

$$
\sup _{x \in \Omega} m(x) \leq 1
$$

Step 1: Let $\mu_{1}(\lambda)$ and $\varphi_{1}(x)$ be the first eigenvalue and associated eigenfunction of the Fréchet derivative $F_{u}(\lambda, u(\lambda))$, that is,

$$
\begin{cases}(-\Delta-\lambda m(x)+2 \lambda h(x) u(\lambda)) \varphi_{1}=\mu_{1}(\lambda) \varphi_{1} & \text { in } \Omega, \\ \varphi_{1}=0 & \text { on } \partial \Omega .\end{cases}
$$

We recall that $\mu_{1}(\lambda)>0$ and $\varphi_{1}(x)>0$ in $\Omega$. Moreover, by Rayleigh's theorem we know that the first eigenvalue $\mu_{1}(\lambda)$ can be characterized by the following formula:

$$
\begin{aligned}
\mu_{1}(\lambda) \int_{\Omega} \varphi^{2} d x \leq & \int_{\Omega}|\nabla \varphi|^{2} d x-\lambda \int_{\Omega} m(x) \varphi^{2} d x \\
& +2 \lambda \int_{\Omega} h(x) u(\lambda) \varphi^{2} d x, \quad \varphi \in W_{0}^{1,2}(\Omega) .
\end{aligned}
$$

Now, we take

$$
\varphi=\left(h^{*}\right)^{s} u(\lambda)^{k}, \quad s>0, k>2,
$$

where the constants $s, k$ will be chosen later on. Then we have

$$
\nabla \varphi=s\left(h^{*}\right)^{s-1} u(\lambda)^{k} \nabla h^{*}+k\left(h^{*}\right)^{s} u(\lambda)^{k-1} \nabla u(\lambda)
$$

and so

$$
\begin{aligned}
|\nabla \varphi|^{2}= & s^{2}\left(h^{*}\right)^{2 s-2} u(\lambda)^{2 k}\left|\nabla h^{*}\right|^{2}+k^{2}\left(h^{*}\right)^{2 s} u(\lambda)^{2 k-2}|\nabla u(\lambda)|^{2} \\
& +2 s k\left(h^{*}\right)^{2 s-1} u(\lambda)^{2 k-1} \nabla h^{*} \cdot \nabla u(\lambda) .
\end{aligned}
$$

Hence we can write inequality (3.4) in the following form:

$$
\begin{aligned}
\mu_{1}(\lambda) \int_{\Omega}\left(h^{*}\right)^{2 s} u(\lambda)^{2 k} d x \leq & s^{2} \int_{\Omega}\left(h^{*}\right)^{2 s-2} u(\lambda)^{2 k}\left|\nabla h^{*}\right|^{2} d x \\
& +2 s k \int_{\Omega}\left(h^{*}\right)^{2 s-1} u(\lambda)^{2 k-1} \nabla h^{*} \cdot \nabla u(\lambda) d x \\
& +k^{2} \int_{\Omega}\left(h^{*}\right)^{2 s} u(\lambda)^{2 k-2}|\nabla u(\lambda)|^{2} d x \\
& -\lambda \int_{\Omega} m(x)\left(h^{*}\right)^{2 s} u(\lambda)^{2 k} d x
\end{aligned}
$$




$$
+2 \lambda \int_{\Omega} h(x)\left(h^{*}\right)^{2 s} u(\lambda)^{2 k+1} d x .
$$

Step 2: Next we show that the second term on the right-hand side of inequality (3.5) can be written as

$$
\begin{aligned}
& 2 s k \int_{\Omega}\left(h^{*}\right)^{2 s-1} u(\lambda)^{2 k-1} \nabla h^{*} \cdot \nabla u(\lambda) d x \\
= & \lambda k \int_{\Omega} m(x)\left(h^{*}\right)^{2 s} u(\lambda)^{2 k} d x \\
& -k(2 k-1) \int_{\Omega}\left(h^{*}\right)^{2 s} u(\lambda)^{2 k-2}|\nabla u(\lambda)|^{2} d x \\
& -\lambda k \int_{\Omega} h(x)\left(h^{*}\right)^{2 s} u(\lambda)^{2 k+1} d x .
\end{aligned}
$$

If we let

$$
\psi(\lambda)=k u(\lambda)^{2 k-1}\left(h^{*}\right)^{2 s},
$$

then we obtain that

$$
\nabla \psi(\lambda)=2 s k u(\lambda)^{2 k-1}\left(h^{*}\right)^{2 s-1} \nabla h^{*}+k(2 k-1) u(\lambda)^{2 k-2}\left(h^{*}\right)^{2 s} \nabla u(\lambda) .
$$

Recall that the function $u(\lambda)$ is a solution of problem (1.6). Hence we have, by Green's formula,

$$
\begin{aligned}
0= & \int_{\Omega}\left(-\Delta u(\lambda)-\lambda m(x) u(\lambda)+\lambda h(x) u(\lambda)^{2}\right) \psi(\lambda) d x \\
= & \int_{\Omega} \nabla u(\lambda) \cdot \nabla \psi(\lambda) d x-\lambda \int_{\Omega} m(x) u(\lambda) \psi(\lambda) d x \\
& +\lambda \int_{\Omega} h(x) u(\lambda)^{2} \psi(\lambda) d x \\
= & \int_{\Omega} \nabla u(\lambda) \cdot\left(2 s k u(\lambda)^{2 k-1}\left(h^{*}\right)^{2 s-1} \nabla h^{*}\right. \\
& \left.+k(2 k-1) u(\lambda)^{2 k-2}\left(h^{*}\right)^{2 s} \nabla u(\lambda)\right) d x \\
& -\lambda \int_{\Omega} m(x) u(\lambda) \psi(\lambda) d x+\lambda \int_{\Omega} h(x) u(\lambda)^{2} \psi(\lambda) d x
\end{aligned}
$$

This proves formula (3.6).

Thus, carrying formula (3.6) into inequality (3.5) we find that

$$
\mu_{1}(\lambda) \int_{\Omega}\left(h^{*}\right)^{2 s} u(\lambda)^{2 k} d x \leq s^{2} \int_{\Omega}\left(h^{*}\right)^{2 s-2} u(\lambda)^{2 k}\left|\nabla h^{*}\right|^{2} d x
$$




$$
\begin{aligned}
& +\lambda(k-1) \int_{\Omega} m(x)\left(h^{*}\right)^{2 s} u(\lambda)^{2 k} d x \\
& -k(k-1) \int_{\Omega}\left(h^{*}\right)^{2 s} u(\lambda)^{2 k-2}|\nabla u(\lambda)|^{2} d x \\
& +\lambda(2-k) \int_{\Omega} h(x)\left(h^{*}\right)^{2 s} u(\lambda)^{2 k+1} d x .
\end{aligned}
$$

In particular, this proves that

$$
\begin{aligned}
& s^{2} \int_{\Omega}\left(h^{*}\right)^{2 s-2} u(\lambda)^{2 k}\left|\nabla h^{*}\right|^{2} d x+\lambda(k-1) \int_{\Omega} m(x)\left(h^{*}\right)^{2 s} u(\lambda)^{2 k} d x \\
= & s^{2} \int_{\Omega^{+}(h)}\left(h^{*}\right)^{2 s-2} u(\lambda)^{2 k}\left|\nabla h^{*}\right|^{2} d x \\
& +\lambda(k-1) \int_{\Omega^{+}(h)} m(x)\left(h^{*}\right)^{2 s} u(\lambda)^{2 k} d x \\
\geq & k(k-1) \int_{\Omega^{+}(h)}\left(h^{*}\right)^{2 s} u(\lambda)^{2 k-2}|\nabla u(\lambda)|^{2} d x \\
& +\lambda(k-2) \int_{\Omega^{+}(h)} h(x)\left(h^{*}\right)^{2 s} u(\lambda)^{2 k+1} d x \\
\geq & k(k-1) \int_{\Omega^{+}(h)}\left(h^{*}\right)^{2 s} u(\lambda)^{2 k-2}|\nabla u(\lambda)|^{2} d x \\
& +\lambda(k-2) \int_{\Omega^{+}(h)}\left(h^{*}\right)^{2 s+1} u(\lambda)^{2 k+1} d x,
\end{aligned}
$$

since $k>2, \mu_{1}(\lambda)>0$ and also we have, by part (a) of Lemma 3.1, $0<h^{*}(x) \leq$ $h(x)$ for $x \in \Omega^{+}(h)$.

Step 3: First, we show that the first term on the left-hand side of inequality (3.7) can be estimated as follows:

$$
\begin{aligned}
& s^{2} \int_{\Omega}\left(h^{*}\right)^{2 s-2} u(\lambda)^{2 k}\left|\nabla h^{*}\right|^{2} d x \\
= & s^{2} \int_{\Omega^{+}(h)}\left(h^{*}\right)^{2 s-2} u(\lambda)^{2 k}\left|\nabla h^{*}\right|^{2} d x \\
\leq & \int_{\Omega^{+}(h)}\left(h^{*}\right)^{1+2 s} u(\lambda)^{2 k+1} d x+\left|\Omega^{+}(h)\right|\left(\frac{C^{2} s^{2}}{\varepsilon^{4}}\right)^{2 k+1} .
\end{aligned}
$$

By inequality (3.2), it follows that

$$
\int_{\Omega}\left(h^{*}\right)^{2 s-2} u(\lambda)^{2 k}\left|\nabla h^{*}\right|^{2} d x=\int_{\Omega^{+}(h)}\left(h^{*}\right)^{2(s-\varepsilon)} u(\lambda)^{2 k} \frac{\left|\nabla h^{*}\right|^{2}}{\left|\left(h^{*}\right)^{1-\varepsilon}\right|^{2}} d x
$$




$$
\leq \frac{C^{2}}{\varepsilon^{4}} \int_{\Omega^{+}(h)}\left(h^{*}\right)^{2(s-\varepsilon)} u(\lambda)^{2 k} d x .
$$

If we choose the constant $s$ as

$$
s=(1+2 \varepsilon) k+\varepsilon,
$$

then we obtain from Hölder's inequality that

$$
\begin{aligned}
& \int_{\Omega}\left(h^{*}\right)^{2 s-2} u(\lambda)^{2 k}\left|\nabla h^{*}\right|^{2} d x \\
\leq & \frac{C^{2}}{\varepsilon^{4}}\left(\int_{\Omega^{+}(h)}\left(\left(h^{*}\right)^{2(s-\varepsilon)} u(\lambda)^{2 k}\right)^{\frac{2 k+1}{2 k}} d x\right)^{\frac{2 k}{2 k+1}}\left(\int_{\Omega^{+}(h)} d x\right)^{\frac{1}{2 k+1}} \\
= & \frac{C^{2}}{\varepsilon^{4}}\left(\int_{\Omega^{+}(h)}\left(h^{*}\right)^{1+2 s} u(\lambda)^{2 k+1} d x\right)^{\frac{2 k}{2 k+1}}\left|\Omega^{+}(h)\right|^{\frac{1}{2 k+1}} .
\end{aligned}
$$

Hence it follows from an application of Young's inequality that

$$
\begin{aligned}
& s^{2} \int_{\Omega^{+}(h)}\left(h^{*}\right)^{2 s-2} u(\lambda)^{2 k}\left|\nabla h^{*}\right|^{2} d x \\
\leq & \left(\int_{\Omega^{+}(h)}\left(h^{*}\right)^{1+2 s} u(\lambda)^{2 k+1} d x\right)^{\frac{2 k}{2 k+1}}\left(\left|\Omega^{+}(h)\right|\left(\frac{C^{2} s^{2}}{\varepsilon^{4}}\right)^{2 k+1}\right)^{\frac{1}{2 k+1}} \\
\leq & \left(\frac{2 k}{2 k+1}\right) \int_{\Omega^{+}(h)}\left(h^{*}\right)^{1+2 s} u(\lambda)^{2 k+1} d x \\
& +\left(\frac{1}{2 k+1}\right)\left|\Omega^{+}(h)\right|\left(\frac{C^{2} s^{2}}{\varepsilon^{4}}\right)^{2 k+1} \\
\leq & \int_{\Omega^{+}(h)}\left(h^{*}\right)^{1+2 s} u(\lambda)^{2 k+1} d x+\left|\Omega^{+}(h)\right|\left(\frac{C^{2} s^{2}}{\varepsilon^{4}}\right)^{2 k+1} .
\end{aligned}
$$

This proves inequality (3.8).

Next we show that the second term on the left-hand side of inequality (3.7) can be estimated as follows:

$$
\begin{aligned}
& \lambda(k-1) \int_{\Omega} m(x)\left(h^{*}\right)^{2 s} u(\lambda)^{2 k} d x \\
= & \lambda(k-1) \int_{\Omega^{+}(h)} m(x)\left(h^{*}\right)^{2 s} u(\lambda)^{2 k} d x \\
\leq & \int_{\Omega^{+}(h)}\left(h^{*}\right)^{1+2 s} u(\lambda)^{2 k+1} d x
\end{aligned}
$$




$$
+\left(\lambda(k-1) \sup _{\Omega^{+}(h)}\left|\left(h^{*}\right)^{2 \varepsilon}\right|\right)^{2 k+1}\left|\Omega^{+}(h)\right| .
$$

Since $\sup _{\Omega} m(x) \leq 1$, it follows from an application of Hölder's and Young's inequalities that

$$
\begin{aligned}
& \lambda(k-1) \int_{\Omega^{+}(h)} m(x)\left(h^{*}\right)^{2 s} u(\lambda)^{2 k} d x \\
& =\lambda(k-1) \int_{\Omega^{+}(h)} m(x)\left(h^{*}\right)^{2(s-\varepsilon)}\left(h^{*}\right)^{2 \varepsilon} u(\lambda)^{2 k} d x \\
& \leq \lambda(k-1) \sup _{\Omega^{+}(h)}\left|\left(h^{*}\right)^{2 \varepsilon}\right| \int_{\Omega^{+}(h)}\left(h^{*}\right)^{2(s-\varepsilon)} u(\lambda)^{2 k} d x \\
& \leq \lambda(k-1) \sup _{\Omega^{+}(h)}\left|\left(h^{*}\right)^{2 \varepsilon}\right|\left(\int_{\Omega^{+}(h)}\left(h^{*}\right)^{1+2 s} u(\lambda)^{2 k+1} d x\right)^{\frac{2 k}{2 k+1}}\left|\Omega^{+}(h)\right|^{\frac{1}{2 k+1}} \\
& =\left(\left(\lambda(k-1) \sup _{\Omega^{+}(h)}\left|\left(h^{*}\right)^{2 \varepsilon}\right|\right)^{2 k+1}\left|\Omega^{+}(h)\right|\right)^{\frac{1}{2 k+1}} \\
& \times\left(\int_{\Omega^{+}(h)}\left(h^{*}\right)^{1+2 s} u(\lambda)^{2 k+1} d x\right)^{\frac{2 k}{2 k+1}} \\
& \leq\left(\frac{1}{2 k+1}\right)\left(\lambda(k-1) \sup _{\Omega^{+}(h)}\left|\left(h^{*}\right)^{2 \varepsilon}\right|\right)^{2 k+1}\left|\Omega^{+}(h)\right| \\
& +\left(\frac{2 k}{2 k+1}\right) \int_{\Omega^{+}(h)}\left(h^{*}\right)^{1+2 s} u(\lambda)^{2 k+1} d x \\
& \leq\left(\lambda(k-1) \sup _{\Omega^{+}(h)}\left|\left(h^{*}\right)^{2 \varepsilon}\right|\right)^{2 k+1}\left|\Omega^{+}(h)\right| \\
& +\int_{\Omega^{+}(h)}\left(h^{*}\right)^{1+2 s} u(\lambda)^{2 k+1} d x .
\end{aligned}
$$

This proves inequality (3.10).

Therefore, combining inequalities (3.7), (3.8) and (3.10) we obtain that

$$
\begin{aligned}
& \quad k(k-1) \int_{\Omega^{+}(h)}\left(h^{*}\right)^{2 s} u(\lambda)^{2 k-2}|\nabla u(\lambda)|^{2} d x \\
& \quad+\lambda(k-2) \int_{\Omega^{+}(h)}\left(h^{*}\right)^{1+2 s} u(\lambda)^{2 k+1} d x \\
& \leq 2 \int_{\Omega^{+}(h)}\left(h^{*}\right)^{1+2 s} u(\lambda)^{2 k+1} d x+\left(\frac{C^{2} s^{2}}{\varepsilon^{4}}\right)^{2 k+1}\left|\Omega^{+}(h)\right|
\end{aligned}
$$




$$
+\left(\lambda(k-1) \sup _{\Omega^{+}(h)}\left|\left(h^{*}\right)^{2 \varepsilon}\right|\right)^{2 k+1}\left|\Omega^{+}(h)\right| .
$$

In particular, this proves that

$$
\begin{aligned}
& \lambda(k-2) \int_{\Omega^{+}(h)}\left(h^{*}\right)^{1+2 s} u(\lambda)^{2 k+1} d x \\
& \leq 2 \int_{\Omega^{+}(h)}\left(h^{*}\right)^{1+2 s} u(\lambda)^{2 k+1} d x+\left(\frac{C^{2} s^{2}}{\varepsilon^{4}}\right)^{2 k+1}\left|\Omega^{+}(h)\right| \\
& \quad+\left(\lambda(k-1) \sup _{\Omega^{+}(h)}\left|\left(h^{*}\right)^{2 \varepsilon}\right|\right)^{2 k+1}\left|\Omega^{+}(h)\right| .
\end{aligned}
$$

If we take the constant $k$ so large that the first term on the right-hand side of inequality (3.11) may be absorbed into the left-hand side, for example, if we take $k$ so large that

$$
k \geq 2+\frac{3}{\lambda}
$$

then it follows that

$$
\begin{aligned}
& \int_{\Omega^{+}(h)}\left(h^{*}\right)^{1+2 s} u(\lambda)^{2 k+1} d x \\
\leq & \left(\left(\frac{C^{2} s^{2}}{\varepsilon^{4}}\right)^{2 k+1}+\left(\lambda(k-1) \sup _{\Omega^{+}(h)}\left|\left(h^{*}\right)^{2 \varepsilon}\right|\right)^{2 k+1}\right)\left|\Omega^{+}(h)\right| .
\end{aligned}
$$

However, by formula (3.9) we find that the constant $s$ is of order $k$. Thus we can find a constant $C^{\prime}>0$ such that

$$
\int_{\Omega^{+}(h)}\left(h^{*}\right)^{1+2 s} u(\lambda)^{2 k+1} d x \leq\left(C^{\prime}(1+\lambda) \frac{k^{2}}{\varepsilon^{4}}\right)^{2 k+1}\left|\Omega^{+}(h)\right| .
$$

Here we remark that the constant $C^{\prime}>0$ depends on the quantities $\sup _{\Omega^{+}(h)}\left|h^{*}\right|$ and $\sup _{\Omega^{+}(h)}|\nabla h|$.

On the other hand, since we have, by formula (3.9),

$$
1+2 s=(1+2 \varepsilon)(2 k+1)
$$

we can write the left-hand side of inequality (3.12) as

$$
\int_{\Omega^{+}(h)}\left(h^{*}\right)^{1+2 s} u(\lambda)^{2 k+1} d x=\int_{\Omega^{+}(h)}\left(\left(h^{*}\right)^{1+2 \varepsilon} u(\lambda)\right)^{2 k+1} d x .
$$


Therefore, we obtain from inequality (3.12) that

$$
\int_{\Omega^{+}(h)}\left(\left(h^{*}\right)^{1+2 \varepsilon} u(\lambda)\right)^{2 k+1} d x \leq\left(C^{\prime}(1+\lambda) \frac{k^{2}}{\varepsilon^{4}}\right)^{2 k+1}\left|\Omega^{+}(h)\right| .
$$

Step 4: We let

$$
\omega(\lambda)=\left(h^{*}\right)^{\frac{s}{k}} u(\lambda)
$$

where (see formula (3.9))

$$
\frac{s}{k}=1+2 \varepsilon+\frac{\varepsilon}{k}
$$

Then we have

$$
\nabla \omega(\lambda)^{k}=s\left(h^{*}\right)^{s-1} u(\lambda)^{k} \nabla h^{*}+k\left(h^{*}\right)^{s} u(\lambda)^{k-1} \nabla u(\lambda)
$$

and so

$$
\begin{aligned}
\left|\nabla \omega(\lambda)^{k}\right|^{2}= & s^{2}\left(h^{*}\right)^{2 s-2} u(\lambda)^{2 k}\left|\nabla h^{*}\right|^{2}+k^{2}\left(h^{*}\right)^{2 s} u(\lambda)^{2 k-2}|\nabla u(\lambda)|^{2} \\
& +2 s k\left(h^{*}\right)^{2 s-1} u(\lambda)^{2 k-1} \nabla h^{*} \cdot \nabla u(\lambda) \\
\leq & 2\left(s^{2}\left(h^{*}\right)^{2 s-2} u(\lambda)^{2 k}\left|\nabla h^{*}\right|^{2}+k^{2}\left(h^{*}\right)^{2 s} u(\lambda)^{2 k-2}|\nabla u(\lambda)|^{2}\right) .
\end{aligned}
$$

Hence it follows that

$$
\begin{aligned}
\int_{\Omega^{+}(h)}\left|\nabla \omega(\lambda)^{k}\right|^{2} d x \leq & 2 s^{2} \int_{\Omega^{+}(h)}\left(h^{*}\right)^{2 s-2} u(\lambda)^{2 k}\left|\nabla h^{*}\right|^{2} d x \\
& +2 k^{2} \int_{\Omega^{+}(h)}\left(h^{*}\right)^{2 s} u(\lambda)^{2 k-2}|\nabla u(\lambda)|^{2} d x .
\end{aligned}
$$

On the other hand, we find from inequality (3.7) that

$$
\begin{aligned}
& \int_{\Omega^{+}(h)}\left(h^{*}\right)^{2 s} u(\lambda)^{2 k-2}|\nabla u(\lambda)|^{2} d x \\
\leq & \frac{s^{2}}{k(k-1)} \int_{\Omega^{+}(h)}\left(h^{*}\right)^{2 s-2} u(\lambda)^{2 k}\left|\nabla h^{*}\right|^{2} d x \\
& +\frac{\lambda}{k} \int_{\Omega^{+}(h)} m(x)\left(h^{*}\right)^{2 s} u(\lambda)^{2 k} d x .
\end{aligned}
$$

Thus, combining inequalities (3.14) and (3.15) we obtain that

$$
\int_{\Omega^{+}(h)}\left|\nabla \omega(\lambda)^{k}\right|^{2} d x
$$




$$
\begin{aligned}
\leq & 2 s^{2}\left(\frac{2 k-1}{k-1}\right) \int_{\Omega^{+}(h)}\left(h^{*}\right)^{2 s-2} u(\lambda)^{2 k}\left|\nabla h^{*}\right|^{2} d x \\
& +2 k \lambda \int_{\Omega^{+}(h)} m(x)\left(h^{*}\right)^{2 s} u(\lambda)^{2 k} d x .
\end{aligned}
$$

However, we recall that the two terms on the right-hand side of inequality (3.16) can be estimated respectively as follows:

$$
\begin{aligned}
& \int_{\Omega^{+}(h)}\left(h^{*}\right)^{2 s-2} u(\lambda)^{2 k}\left|\nabla h^{*}\right|^{2} d x \leq \frac{C^{2}}{\varepsilon^{4}} \int_{\Omega^{+}(h)}\left(h^{*}\right)^{2(s-\varepsilon)} u(\lambda)^{2 k} d x . \\
& \int_{\Omega^{+}(h)}\left(h^{*}\right)^{2 s} u(\lambda)^{2 k} d x \leq \sup _{\Omega^{+}(h)}\left|\left(h^{*}\right)^{2 \varepsilon}\right| \int_{\Omega^{+}(h)}\left(h^{*}\right)^{2(s-\varepsilon)} u(\lambda)^{2 k} d x .
\end{aligned}
$$

Therefore, carrying these inequalities into the right-hand side of inequality (3.16) we obtain that

$$
\begin{aligned}
& \int_{\Omega^{+}(h)}\left|\nabla \omega(\lambda)^{k}\right|^{2} d x \\
\leq & \left(2 s^{2}\left(\frac{2 k-1}{k-1}\right) \frac{C^{2}}{\varepsilon^{4}}+2 \lambda k \sup _{\Omega^{+}(h)}\left|\left(h^{*}\right)^{2 \varepsilon}\right|\right) \int_{\Omega^{+}(h)}\left(h^{*}\right)^{2(s-\varepsilon)} u(\lambda)^{2 k} d x .
\end{aligned}
$$

However, by formula (3.9) we find that the constant $s$ is of order $k$. Thus, if we take the constant $k$ so large that

$$
\frac{2 k-1}{k-1}<3
$$

then we can find a constant $C^{\prime \prime}>0$ such that

$$
\begin{aligned}
& \int_{\Omega^{+}(h)}\left|\nabla \omega(\lambda)^{k}\right|^{2} d x \\
\leq & \left(6 s^{2} \frac{C^{2}}{\varepsilon^{4}}+2 \lambda k \sup _{\Omega^{+}(h)}\left|\left(h^{*}\right)^{2 \varepsilon}\right|\right) \int_{\Omega^{+}(h)}\left(h^{*}\right)^{2(s-\varepsilon)} u(\lambda)^{2 k} d x \\
\leq & C^{\prime \prime}(1+\lambda) \frac{k^{2}}{\varepsilon^{4}} \int_{\Omega^{+}(h)}\left(h^{*}\right)^{2(s-\varepsilon)} u(\lambda)^{2 k} d x .
\end{aligned}
$$

Here we remark that the constant $C^{\prime \prime}>0$ depends on the quantities $\sup _{\Omega^{+}(h)}\left|h^{*}\right|$ and $\sup _{\Omega^{+}(h)}|\nabla h|$.

Step 5: We make use of the Sobolev imbedding theorem (cf. Adams-Fournier [1, Theorem 4.12])

$$
\left(\int_{\Omega^{+}(h)} \varphi^{\frac{2 n}{n-2}} d x\right)^{\frac{n-2}{2 n}} \leq C(n)\left(\int_{\Omega^{+}(h)}|\nabla \varphi|^{2} d x\right)^{\frac{1}{2}}, \quad \varphi \in W^{1,2}\left(\Omega^{+}(h)\right) .
$$


Here the constant $C(n)>0$ depends on the dimension $n \geq 3$.

Now, applying inequality (3.18) to the function

$$
\omega(\lambda)^{k}=\left(h^{*}\right)^{s} u(\lambda)^{k}
$$

and then using inequality (3.17), we obtain that

$$
\begin{aligned}
& \left(\int_{\Omega^{+}(h)}\left(\left(h^{*}\right)^{\frac{s}{k}} u(\lambda)\right)^{\frac{2 k n}{n-2}} d x\right)^{\frac{n-2}{n}} \\
= & \left(\int_{\Omega^{+}(h)}\left(\left(h^{*}\right)^{s} u(\lambda)^{k}\right)^{\frac{2 n}{n-2}} d x\right)^{\frac{n-2}{n}} \\
\leq & C(n)^{2} \int_{\Omega^{+}(h)}\left|\nabla \omega(\lambda)^{k}\right|^{2} d x \\
\leq & C(n)^{2} C^{\prime \prime}(1+\lambda) \frac{k^{2}}{\varepsilon^{4}} \int_{\Omega^{+}(h)}\left(h^{*}\right)^{2(s-\varepsilon)} u(\lambda)^{2 k} d x \\
= & C(n)^{2} C^{\prime \prime}(1+\lambda) \frac{k^{2}}{\varepsilon^{4}} \int_{\Omega^{+}(h)}\left(\left(h^{*}\right)^{\frac{s-\varepsilon}{k}} u(\lambda)\right)^{2 k} d x,
\end{aligned}
$$

or equivalently

$$
\begin{aligned}
& \left\|\left(h^{*}\right)^{s / k} u(\lambda)\right\|_{L^{2 k n /(n-2)}\left(\Omega^{+}(h)\right)} \\
\leq & C(\lambda)^{1 / 2 k}\left(\frac{k^{2}}{\varepsilon^{4}}\right)^{1 / 2 k}\left\|\left(h^{*}\right)^{(s-\varepsilon) / k} u(\lambda)\right\|_{L^{2 k}\left(\Omega^{+}(h)\right)},
\end{aligned}
$$

where

$$
C(\lambda)=C(n)^{2} C^{\prime \prime}(1+\lambda)
$$

We let

$$
\chi=\frac{n}{n-2}>1
$$

and, for a sufficiently large positive integer $\ell$,

$$
k=\chi^{\ell}
$$

Then we have

$$
\frac{s}{k}=1+2 \varepsilon+\frac{\varepsilon}{k}=1+2 \varepsilon+\frac{\varepsilon}{\chi^{\ell}},
$$




$$
\begin{aligned}
& \frac{2 k n}{n-2}=2 \chi^{\ell} \frac{n}{n-2}=2 \chi^{\ell+1}, \\
& \frac{s-\varepsilon}{k}=1+2 \varepsilon .
\end{aligned}
$$

Thus we can write inequality (3.19) as

$$
\begin{aligned}
& \left\|\left(h^{*}\right)^{1+2 \varepsilon+\frac{\varepsilon}{\chi^{\ell}} u(\lambda)}\right\|_{L^{2 \chi^{\ell+1}}\left(\Omega^{+}(h)\right)} \\
\leq & C(\lambda)^{\frac{1}{2 \chi^{\ell}}}\left(\frac{\chi^{2 \ell}}{\varepsilon^{4}}\right)^{\frac{1}{2 \chi^{\ell}}}\left\|\left(h^{*}\right)^{1+2 \varepsilon} u(\lambda)\right\|_{L^{2 \chi^{\ell}}\left(\Omega^{+}(h)\right)} .
\end{aligned}
$$

Furthermore, if we let

$$
\varepsilon_{0}=\varepsilon, \quad \varepsilon_{1}=\left(1+\frac{1}{2 \chi^{\ell}}\right) \varepsilon_{0}
$$

then it follows that

$$
1+2 \varepsilon_{0}+\frac{\varepsilon_{0}}{\chi^{\ell}}=1+2\left(1+\frac{1}{2 \chi^{\ell}}\right) \varepsilon_{0}=1+2 \varepsilon_{1} .
$$

Thus we can rewrite inequality (3.20) in the following form:

$$
\begin{aligned}
& \left\|\left(h^{*}\right)^{1+2 \varepsilon_{1}} u(\lambda)\right\|_{L^{2 \chi^{\ell+1}}\left(\Omega^{+}(h)\right)} \\
\leq & C(\lambda)^{\frac{1}{2} \chi^{-\ell}}\left(\frac{\chi^{2 \ell}}{\varepsilon_{0}^{4}}\right)^{\frac{1}{2} \chi^{-\ell}}\left\|\left(h^{*}\right)^{1+2 \varepsilon_{0}} u(\lambda)\right\|_{L^{2 \chi^{\ell}}\left(\Omega^{+}(h)\right)} .
\end{aligned}
$$

Step 6: By the same procedure as above (replacing $\chi^{\ell}$ by $\chi^{\ell+1}$ ), we have the inequality

$$
\begin{aligned}
& \left\|\left(h^{*}\right)^{1+2 \varepsilon_{1}+\frac{\varepsilon_{1}}{\chi^{\ell+1}} u(\lambda)}\right\|_{L^{2 \chi^{\ell+2}\left(\Omega^{+}(h)\right)}} \\
\leq & C(\lambda)^{\frac{1}{2} \chi^{-(\ell+1)}}\left(\frac{\chi^{2(\ell+1)}}{\varepsilon_{1}^{4}}\right)^{\frac{1}{2} \chi^{-(\ell+1)}}\left\|\left(h^{*}\right)^{1+2 \varepsilon_{1}} u(\lambda)\right\|_{L^{2 \chi^{\ell+1}}\left(\Omega^{+}(h)\right)} .
\end{aligned}
$$

However, we remark that

$$
\varepsilon_{1}=\left(1+\frac{1}{2 \chi^{\ell}}\right) \varepsilon_{0}>\varepsilon_{0} .
$$

Thus, combining inequality (3.22) with inequality $(3.21)_{1}$ we obtain that

$$
\left\|\left(h^{*}\right)^{1+2 \varepsilon_{1}+\frac{\varepsilon_{1}}{\chi^{\ell+1}} u(\lambda)}\right\|_{L^{2 \chi^{\ell+2}}\left(\Omega^{+}(h)\right)}
$$




$$
\begin{aligned}
\leq & C(\lambda)^{\frac{1}{2} \chi^{-(\ell+1)}}\left(\frac{\chi^{2(\ell+1)}}{\varepsilon_{0}^{4}}\right)^{\frac{1}{2} \chi^{-(\ell+1)}}\left\|\left(h^{*}\right)^{1+2 \varepsilon_{1}} u(\lambda)\right\|_{L^{2 \chi^{\ell+1}}\left(\Omega^{+}(h)\right)} \\
\leq & C(\lambda)^{\frac{1}{2} \chi^{-(\ell+1)}} C(\lambda)^{\frac{1}{2} \chi^{-\ell}}\left(\frac{\chi^{2(\ell+1)}}{\varepsilon_{0}^{4}}\right)^{\frac{1}{2} \chi^{-(\ell+1)}}\left(\frac{\chi^{2 \ell}}{\varepsilon_{0}^{4}}\right)^{\frac{1}{2} \chi^{-\ell}} \\
& \times\left\|\left(h^{*}\right)^{1+2 \varepsilon_{0}} u(\lambda)\right\|_{L^{2 \chi^{\ell}}\left(\Omega^{+}(h)\right)} \\
\leq & C(\lambda)^{\frac{1}{2}\left(\chi^{-(\ell+1)}+\chi^{-\ell}\right)} \chi^{\left((\ell+1) \chi^{-(\ell+1)}+\ell \chi^{-\ell}\right)}\left(\frac{1}{\varepsilon_{0}}\right)^{2\left(\chi^{-(\ell+1)}+\chi^{-\ell}\right)} \\
& \times\left\|\left(h^{*}\right)^{1+2 \varepsilon_{0}} u(\lambda)\right\|_{L^{2 \chi^{\ell}}\left(\Omega^{+}(h)\right)}
\end{aligned}
$$

If we let

$$
\varepsilon_{2}=\left(1+\frac{1}{2 \chi^{\ell+1}}\right) \varepsilon_{1}
$$

then we can write inequality (3.23) as

$$
\begin{aligned}
& \left\|\left(h^{*}\right)^{1+2 \varepsilon_{2}} u(\lambda)\right\|_{L^{2 \chi^{\ell+2}}\left(\Omega^{+}(h)\right)} \\
\leq & C(\lambda)^{\frac{1}{2}\left(\chi^{-\ell}+\chi^{-(\ell+1)}\right)} \chi^{\left(\ell \chi^{-\ell}+(\ell+1) \chi^{-(\ell+1)}\right)}\left(\frac{1}{\varepsilon_{0}}\right)^{2\left(\chi^{-\ell}+\chi^{-(\ell+1)}\right)} \\
& \times\left\|\left(h^{*}\right)^{1+2 \varepsilon_{0}} u(\lambda)\right\|_{L^{2 \chi^{\ell}}\left(\Omega^{+}(h)\right)}
\end{aligned}
$$

Continuing this procedure, we have, after $N$ steps,

$$
\begin{aligned}
& \left\|\left(h^{*}\right)^{1+2 \varepsilon_{N+1}} u(\lambda)\right\|_{L^{2 \chi^{\ell+N+1}}\left(\Omega^{+}(h)\right)} \\
\leq & C(\lambda)^{\frac{1}{2}\left(\sum_{i=0}^{N} \chi^{-(\ell+i)}\right)} \chi^{\left(\sum_{i=0}^{N}(\ell+i) \chi^{-(\ell+i)}\right)}\left(\frac{1}{\varepsilon_{0}}\right)^{2\left(\sum_{i=0}^{N} \chi^{-(\ell+i)}\right)} \\
& \times\left\|\left(h^{*}\right)^{1+2 \varepsilon_{0}} u(\lambda)\right\|_{L^{2 \chi^{\ell}}\left(\Omega^{+}(h)\right)}
\end{aligned}
$$

However, we remark that

$$
\begin{aligned}
& \sum_{i=0}^{\infty} \frac{1}{\chi^{\ell+i}}=\frac{1}{\chi^{\ell}} \sum_{i=0}^{\infty} \frac{1}{\chi^{i}}=\frac{1}{\chi^{\ell}}\left(\frac{\chi}{\chi-1}\right)=\frac{n}{2} \frac{1}{\chi^{\ell}}, \\
& \sum_{i=0}^{\infty}(\ell+i) \frac{1}{\chi^{\ell+i}}=\frac{1}{\chi} \sum_{i=0}^{\infty}(\ell+i) \frac{1}{\chi^{\ell+i-1}}=\frac{n(2 \ell+n-2)}{4} \frac{1}{\chi^{\ell}} .
\end{aligned}
$$

Thus it follows from inequality $(3.21)_{N+1}$ that

$$
\left\|\left(h^{*}\right)^{1+2 \varepsilon_{N+1}} u(\lambda)\right\|_{L^{2 \chi^{\ell+N+1}}\left(\Omega^{+}(h)\right)}
$$




$$
\leq C(\lambda)^{\frac{n}{4 \chi^{\ell}}} \chi^{\frac{n(2 \ell+n-2)}{4 \chi^{\ell}}}\left(\frac{1}{\varepsilon_{0}}\right)^{\frac{n}{\chi^{\ell}}}\left\|\left(h^{*}\right)^{1+2 \varepsilon_{0}} u(\lambda)\right\|_{L^{2 \chi^{\ell}\left(\Omega^{+}(h)\right)}} .
$$

Furthermore, we find that

$$
\begin{aligned}
\varepsilon_{N+1} & =\left(1+\frac{1}{2 \chi^{\ell+N}}\right) \varepsilon_{N} \\
& =\left(1+\frac{1}{2 \chi^{\ell+N}}\right)\left(1+\frac{1}{2 \chi^{\ell+N-1}}\right) \varepsilon_{N-1} \\
& \vdots \\
& =\left(\prod_{i=0}^{N}\left(1+\frac{1}{2 \chi^{\ell+i}}\right)\right) \varepsilon_{0},
\end{aligned}
$$

and that the limit (infinite product)

$$
\lim _{N \rightarrow \infty} \varepsilon_{N+1}=\left(\prod_{i=0}^{\infty}\left(1+\frac{1}{2 \chi^{\ell+i}}\right)\right) \varepsilon_{0}
$$

exists, since $\chi>1$.

Therefore, letting $N \rightarrow \infty$ in inequality (3.24), we obtain that

$$
\begin{aligned}
& \sup _{\Omega^{+}(h)}\left|\left(h^{*}\right)^{1+2 \sigma \varepsilon_{0}} u(\lambda)\right| \\
\leq & C(\lambda)^{\frac{n}{4 \chi^{\ell}}} \chi^{\frac{n(2 \ell+n-2)}{4 \chi^{\ell}}}\left(\frac{1}{\varepsilon_{0}}\right)^{\frac{n}{\chi^{\ell}}}\left\|\left(h^{*}\right)^{1+2 \varepsilon_{0}} u(\lambda)\right\|_{L^{2 \chi^{\ell}}\left(\Omega^{+}(h)\right)},
\end{aligned}
$$

where

$$
\sigma=\prod_{i=0}^{\infty}\left(1+\frac{1}{2 \chi^{\ell+i}}\right)>1
$$

Step 7: On the other hand, by Hölder's inequality it follows that

$$
\begin{aligned}
\left\|\left(h^{*}\right)^{1+2 \varepsilon_{0}} u(\lambda)\right\|_{L^{2 \chi^{\ell}}\left(\Omega^{+}(h)\right)} \leq & \left\|\left(h^{*}\right)^{1+2 \varepsilon_{0}} u(\lambda)\right\|_{L^{2 k+1}\left(\Omega^{+}(h)\right)} \\
& \times\left|\Omega^{+}(h)\right|^{\frac{1}{2 \chi^{\ell}}-\frac{1}{2 k+1}} .
\end{aligned}
$$

Furthermore, we have, by inequality (3.13) with $\varepsilon:=\varepsilon_{0}$,

$$
\left\|\left(h^{*}\right)^{1+2 \varepsilon_{0}} u(\lambda)\right\|_{L^{2 k+1}\left(\Omega^{+}(h)\right)} \leq C(\lambda)^{\prime}\left(\frac{k^{2}}{\varepsilon_{0}^{4}}\right)\left|\Omega^{+}(h)\right|^{\frac{1}{2 k+1}}
$$


where

$$
C(\lambda)^{\prime}=C^{\prime}(1+\lambda) .
$$

Here we recall that the constant $C^{\prime}$ depends on the quantities $\sup _{\Omega^{+}(h)}\left|h^{*}\right|$ and $\sup _{\Omega^{+}(h)}|\nabla h|$.

Therefore, combining inequalities (3.26) and (3.27), we get the following inequality:

$$
\left\|\left(h^{*}\right)^{1+2 \varepsilon_{0}} u(\lambda)\right\|_{L^{2 \chi^{\ell}}\left(\Omega^{+}(h)\right)} \leq C(\lambda)^{\prime} \chi^{2 \ell}\left(\frac{1}{\varepsilon_{0}}\right)^{4}\left|\Omega^{+}(h)\right|^{\frac{1}{2 \chi^{\ell}}} .
$$

Carrying this inequality into the right-hand side of inequality (3.25), we obtain that

$$
\begin{aligned}
\sup _{\Omega^{+}(h)}\left|\left(h^{*}\right)^{1+2 \sigma \varepsilon_{0}} u(\lambda)\right| \leq & C(\lambda)^{\frac{n}{4 \chi^{\ell}}} \chi^{\frac{n(2 \ell+n-2)}{4 \chi^{\ell}}} C(\lambda)^{\prime} \chi^{2 \ell}\left(\frac{1}{\varepsilon_{0}}\right)^{\frac{n}{\chi^{\ell}+4}} \\
& \times\left|\Omega^{+}(h)\right|^{\frac{1}{2 \chi^{\ell}}} .
\end{aligned}
$$

Summing up, we have proved that there exists a constant $C(\lambda)^{\prime \prime}>0$ such that, for each $\varepsilon_{0}>0$,

$$
\sup _{\Omega^{+}(h)}\left|\left(h^{*}\right)^{1+2 \sigma \varepsilon_{0}} u(\lambda)\right| \leq C(\lambda)^{\prime \prime} \varepsilon_{0}^{-\mu},
$$

where

$$
\mu=\frac{n}{\chi^{\ell}}+4
$$

It is easy to see that inequality (3.28) is equivalent to inequality (3.3). Moreover, we find that if $\lambda$ is finite, then so is the constant $C(\lambda)^{\prime \prime}$.

The proof of Lemma 3.2 is now complete.

\section{Proof of Theorem $1.2-(3)-$}

The next lemma asserts that the solution $u(\lambda)$ blows up at the critical value $\lambda^{*}$ :

Lemma 4.1. If $u(\lambda) \in C^{2}(\bar{\Omega}), \lambda_{1}(m)<\lambda<\lambda^{*}$, is a solution of problem (1.6), then we have

$$
\lim _{\lambda \rightarrow \lambda^{*}}\|u(\lambda)\|_{L^{2}(\Omega)}=+\infty .
$$

Proof. Assume, to the contrary, that there exists a constant $C>0$ such that

$$
\int_{\Omega} u(\lambda)^{2} d x \leq C \text { for all } \lambda \in\left(\lambda_{1}(m), \lambda^{*}\right) .
$$


Then, using Green's formula we obtain that

$$
\begin{aligned}
0= & \int_{\Omega}\left(-\Delta u(\lambda)-\lambda m(x) u(\lambda)+\lambda h(x) u(\lambda)^{2}\right) u(\lambda) d x \\
= & \int_{\Omega}|\nabla u(\lambda)|^{2} d x-\lambda \int_{\Omega} m(x) u(\lambda)^{2} d x \\
& +\lambda \int_{\Omega} h(x) u(\lambda)^{3} d x .
\end{aligned}
$$

Thus it follows that

$$
\int_{\Omega}|\nabla u(\lambda)|^{2} d x+\lambda \int_{\Omega} h(x) u(\lambda)^{3} d x=\lambda \int_{\Omega} m(x) u(\lambda)^{2} d x .
$$

In particular, this proves that

$$
\int_{\Omega}|\nabla u(\lambda)|^{2} d x \leq \lambda\left\|m^{+}\right\|_{L^{\infty}(\Omega)} \int_{\Omega} u(\lambda)^{2} d x
$$

so that

$$
\int_{\Omega}|\nabla u(\lambda)|^{2} d x \leq \lambda^{*} C\left\|m^{+}\right\|_{L^{\infty}(\Omega)}, \quad \lambda_{1}(m)<\lambda<\lambda^{*} .
$$

Here

$$
m^{+}(x)=\max \{m(x), 0\} .
$$

On the other hand, applying Sobolev's inequality (3.18) to the function $u(\lambda)$ we obtain that

$$
\left(\int_{\Omega} u(\lambda)^{\frac{2 n}{n-2}} d x\right)^{\frac{n-2}{n}} \leq C(n)^{2} \int_{\Omega}|\nabla u(\lambda)|^{2} d x, \quad u \in W^{1,2}(\Omega) .
$$

Thus, combining inequalities (4.4) and (4.3) we obtain that

$$
\left(\int_{\Omega} u(\lambda)^{\frac{2 n}{n-2}} d x\right)^{\frac{n-2}{n}} \leq \lambda C(n)^{2} \int_{\Omega} u(\lambda)^{2} d x
$$

or equivalently

$$
\|u(\lambda)\|_{L^{2 n /(n-2)}(\Omega)} \leq C(\lambda)^{\frac{1}{2}}\|u(\lambda)\|_{L^{2}(\Omega)},
$$

where

$$
C(\lambda)=\lambda C(n)^{2}
$$

Furthermore, if we let

$$
\chi=\frac{n}{n-2}>1 \quad(n \geq 3),
$$


then we can write inequality (4.5) in the following form:

$$
\|u(\lambda)\|_{L^{2 \chi}(\Omega)} \leq C(\lambda)^{\frac{1}{2}}\|u(\lambda)\|_{L^{2}(\Omega)} .
$$

Continuing this procedure as in the proof of Lemma 3.2, we have, after $N$ steps,

$$
\begin{aligned}
\|u(\lambda)\|_{L^{2 \chi^{N+1}}(\Omega)} & \leq C(\lambda)^{\frac{1}{2}\left(\sum_{i=0}^{N} \chi^{-i}\right)}\|u(\lambda)\|_{L^{2}(\Omega)} \\
& \leq C(\lambda)^{\frac{n}{4}}\|u(\lambda)\|_{L^{2}(\Omega)}, \quad \lambda_{1}(m)<\lambda<\lambda^{*} .
\end{aligned}
$$

Therefore, letting $N \rightarrow \infty$ in inequality $(4.6)_{N+1}$ we obtain that

$$
\|u(\lambda)\|_{L^{\infty}(\Omega)} \leq\left(\lambda C(n)^{2}\right)^{\frac{n}{4}} \chi^{\frac{n(n-2)}{4}}\|u(\lambda)\|_{L^{2}(\Omega)}, \quad \lambda_{1}(m)<\lambda<\lambda^{*} .
$$

By inequalities (4.2) and (4.3), it follows that, for all $\lambda_{1}(m)<\lambda<\lambda^{*}$,

$$
\begin{aligned}
& \int_{\Omega} u(\lambda)^{2} d x \leq C, \\
& \int_{\Omega}|\nabla u(\lambda)|^{2} d x \leq \lambda^{*} C\left\|m^{+}\right\|_{L^{\infty}(\Omega)} .
\end{aligned}
$$

This proves that the functions $u(\lambda)$ are bounded in the Sobolev space $W^{1,2}(\Omega)$, for all $\lambda \in\left(\lambda_{1}(m), \lambda^{*}\right)$.

However, we remark the following:

(a) Rellich's theorem tells us that the injection of $W^{1,2}(\Omega)$ into $L^{2}(\Omega)$ is compact (or completely continuous) if the dimension $n$ is greater than $2(n \geq 3)$.

(b) It is well known (see Yosida [27, Chapter V, Section 2, Theorem 1]) that the unit ball in the Hilbert space is sequentially weakly compact. Therefore, by inequalities (4.8a) and (4.8b) we can find a sequence $\left\{\lambda_{n}\right\}$ and a function $u\left(\lambda^{*}\right) \in$ $W^{1,2}(\Omega)$ such that

$$
\lambda_{n} \longrightarrow \lambda^{*}
$$

and that

$$
\begin{aligned}
& u\left(\lambda_{n}\right) \longrightarrow u\left(\lambda^{*}\right) \quad \text { strongly in } L^{2}(\Omega), \\
& \nabla u\left(\lambda_{n}\right) \longrightarrow \nabla u\left(\lambda^{*}\right) \quad \text { weakly in } L^{2}(\Omega) .
\end{aligned}
$$

On the other hand, by combining inequalities (4.2) and (4.7) we obtain that

$$
\sup _{\Omega}|u(\lambda)| \leq C^{\frac{1}{2}}\left(\lambda^{*} C(n)^{2}\right)^{\frac{n}{4}} \chi^{\frac{n(n-2)}{4}} \quad \text { for all } \lambda \in\left(\lambda_{1}(m), \lambda^{*}\right) .
$$


Thus we may assume that the finite limit

$$
u\left(\lambda^{*}\right)(x)=\lim _{\lambda_{n} \rightarrow \lambda^{*}} u\left(\lambda_{n}\right)(x)
$$

exists for almost all $x$ of $\Omega$.

Now, since $u\left(\lambda_{n}\right)$ is a solution of problem (1.6), it follows that, for all $\psi \in$ $W_{0}^{1,2}(\Omega)$,

$$
\int_{\Omega} \nabla u\left(\lambda_{n}\right) \cdot \nabla \psi d x+\lambda_{n} \int_{\Omega} h(x) u\left(\lambda_{n}\right)^{2} \psi d x-\lambda_{n} \int_{\Omega} m(x) u\left(\lambda_{n}\right) \psi d x=0 .
$$

However, we have the following two assertions:

(i) By assertion (4.9), it follows that

$$
\int_{\Omega} u\left(\lambda_{n}\right) \psi d x \longrightarrow \int_{\Omega} u\left(\lambda^{*}\right) \psi d x
$$

and that

$$
\int_{\Omega} \nabla u\left(\lambda_{n}\right) \cdot \nabla \psi d x \longrightarrow \int_{\Omega} \nabla u\left(\lambda^{*}\right) \cdot \nabla \psi d x
$$

(ii) By assertion (4.10), it follows from an application of the Lebesgue bounded convergence theorem that

$$
\int_{\Omega} h(x) u\left(\lambda_{n}\right)^{2} \psi d x \longrightarrow \int_{\Omega} h(x) u\left(\lambda^{*}\right)^{2} \psi d x
$$

By passing to the limit in formula (4.11), we obtain that the function $u\left(\lambda^{*}\right)$ satisfies, for all $\psi \in W_{0}^{1,2}(\Omega)$, the equation

$$
\int_{\Omega} \nabla u\left(\lambda^{*}\right) \cdot \nabla \psi d x+\lambda^{*} \int_{\Omega} h(x) u\left(\lambda^{*}\right)^{2} \psi d x-\lambda^{*} \int_{\Omega} m(x) u\left(\lambda^{*}\right) \psi d x=0 .
$$

This proves that the function $u\left(\lambda^{*}\right) \in W^{1,2}(\Omega)$ is a weak solution of problem (1.6).

Thus it follows from an application of the regularity theorem in quasilinear elliptic theory (see Ladyzhenskaya-Ural'tseva [14, Chapter 4, Theorem 6.5]) that

$$
u\left(\lambda^{*}\right) \in C^{2+\theta}(\bar{\Omega}) .
$$

Furthermore, we recall that the solution $u(\lambda)$ is strictly positive in $\Omega$ and that the continuum $\mathcal{C}$ of positive solutions of problem (1.6) can not contain a point $\left(\lambda_{0}, 0\right)$ with $\lambda_{0} \neq \lambda_{1}(m)$. Thus, by the maximum principle it follows that

$$
u\left(\lambda^{*}\right)>0 \text { in } \Omega \text {. }
$$


Finally, it is easy to see that the Fréchet derivative $F_{u}\left(\lambda^{*}, u\left(\lambda^{*}\right)\right)$ is an algebraic and topological isomorphism. Indeed, if $\mu_{1}\left(\lambda^{*}\right)$ is the first eigenvalue of the Fréchet derivative $F_{u}\left(\lambda^{*}, u\left(\lambda^{*}\right)\right)$, then, arguing as in the proof of Claim 2.6 we obtain that $\mu_{1}\left(\lambda^{*}\right)>0$.

Therefore, by virtue of the implicit function theorem we can extend the bifurcation curve $(\lambda, u(\lambda))$ beyond the point $\left(\lambda^{*}, u\left(\lambda^{*}\right)\right)$. This contradicts the definition of the critical value $\lambda^{*}$.

The proof of Lemma 4.1 is complete.

\section{Proof of Theorem $1.2-(4)-$}

The next proposition proves the inequality

$$
\mu_{1}\left(\Omega_{0}(h)\right) \leq \lambda^{*}
$$

which completes the proof of formula (2.12).

Proposition 5.1. The critical value $\lambda^{*}$ is an eigenvalue of the Dirichlet problem (1.7).

Proof. Step 1: Let $u(\lambda) \in C^{2}(\bar{\Omega}), \lambda_{1}(m)<\lambda<\lambda^{*}$, be a solution of the problem (1.6), and let

$$
\omega(\lambda)=\frac{u(\lambda)}{\|u(\lambda)\|_{L^{2}(\Omega)}}
$$

Then it follows that

$$
\begin{aligned}
& -\Delta \omega(\lambda)-\lambda m(x) \omega(\lambda)+\lambda h(x) u(\lambda) \omega(\lambda) \\
= & \frac{1}{\|u(\lambda)\|_{L^{2}(\Omega)}}\left(-\Delta u(\lambda)-\lambda m(x) u(\lambda)+\lambda h(x) u(\lambda)^{2}\right)=0 \quad \text { in } \Omega,
\end{aligned}
$$

and that

$$
\omega(\lambda)=0 \quad \text { on } \partial \Omega
$$

Hence we have, by Green's formula,

$$
\begin{aligned}
\int_{\Omega} h(x) u(\lambda) \omega(\lambda)^{2} d x & =\int_{\Omega} m(x) \omega(\lambda)^{2} d x+\frac{1}{\lambda} \int_{\Omega} \Delta \omega(\lambda) \cdot \omega(\lambda) d x \\
& =\int_{\Omega} m(x) \omega(\lambda)^{2} d x-\frac{1}{\lambda} \int_{\Omega}|\nabla \omega(\lambda)|^{2} d x
\end{aligned}
$$


This proves that

$$
\begin{aligned}
\frac{1}{\lambda} \int_{\Omega}|\nabla \omega(\lambda)|^{2} d x & \leq \frac{1}{\lambda} \int_{\Omega}|\nabla \omega(\lambda)|^{2} d x+\int_{\Omega} h(x) u(\lambda) \omega(\lambda)^{2} d x \\
& =\int_{\Omega} m(x) \omega(\lambda)^{2} d x \\
& \leq\left\|m^{+}\right\|_{L^{\infty}(\Omega)} \int_{\Omega} \omega(\lambda)^{2} d x \\
& =\left\|m^{+}\right\|_{L^{\infty}(\Omega)} .
\end{aligned}
$$

By inequality (5.1), it follows that, for all $\lambda \in\left(\lambda_{1}(m), \lambda^{*}\right)$,

$$
\begin{aligned}
& \int_{\Omega} \omega(\lambda)^{2} d x=1 \\
& \int_{\Omega}|\nabla \omega(\lambda)|^{2} d x \leq \lambda\left\|m^{+}\right\|_{L^{\infty}(\Omega)} \leq \lambda^{*}\left\|m^{+}\right\|_{L^{\infty}(\Omega)} .
\end{aligned}
$$

Thus, just as in the proof of Lemma 4.1 we can find a sequence $\left\{\lambda_{n}\right\}$ and a function $\omega\left(\lambda^{*}\right) \in W^{1,2}(\Omega)$ such that

$$
\lambda_{n} \longrightarrow \lambda^{*}
$$

and that

$$
\begin{aligned}
& \omega\left(\lambda_{n}\right) \longrightarrow \omega\left(\lambda^{*}\right) \quad \text { strongly in } L^{2}(\Omega), \\
& \nabla \omega\left(\lambda_{n}\right) \longrightarrow \nabla \omega\left(\lambda^{*}\right) \quad \text { weakly in } L^{2}(\Omega) .
\end{aligned}
$$

Furthermore, arguing as in the proof of Lemma 4.1 (see inequality (4.7)) we can find a constant $C\left(\lambda^{*}\right)>0$ such that

$$
\sup _{\Omega}|\omega(\lambda)| \leq C\left(\lambda^{*}\right)\|\omega(\lambda)\|_{L^{2}(\Omega)}=C\left(\lambda^{*}\right) \quad \text { for all } \lambda \in\left(\lambda_{1}(m), \lambda^{*}\right) .
$$

Therefore, we obtain from assertions (5.2), (5.3) and (5.4) that the limit function $\omega\left(\lambda^{*}\right) \in W^{1,2}(\Omega)$ satisfies the following conditions:

$$
\begin{aligned}
& \omega\left(\lambda^{*}\right) \geq 0 \quad \text { in } \Omega . \\
& \int_{\Omega} \omega\left(\lambda^{*}\right)^{2} d x=1 . \\
& \int_{\Omega}\left|\nabla \omega\left(\lambda^{*}\right)\right|^{2} d x \leq \lambda^{*} . \\
& \sup _{\Omega}\left|\omega\left(\lambda^{*}\right)\right| \leq C\left(\lambda^{*}\right) .
\end{aligned}
$$


On the other hand, we remark that the functions $\omega\left(\lambda_{n}\right)$ satisfy the equation

$$
\Delta \omega\left(\lambda_{n}\right)+\lambda_{n} m(x) \omega\left(\lambda_{n}\right)=0 \quad \text { in } \Omega_{0}(h),
$$

since $h \equiv 0$ in $\Omega_{0}(h)$. By passing to the limit, we find that the function $\omega\left(\lambda^{*}\right)$ is a weak solution of the equation

$$
\Delta \omega\left(\lambda^{*}\right)+\lambda^{*} m(x) \omega\left(\lambda^{*}\right)=0 \quad \text { in } \Omega_{0}(h) .
$$

Hence it follows from an application of the interior regularity theorem in linear elliptic theory (see Ladyzhenskaya-Ural'tseva [14, Chapter 3, Theorem 12.1], Gilbarg-Trudinger [11, Corollary 8.11]) that

$$
\omega\left(\lambda^{*}\right) \in C^{2+\theta}\left(\Omega_{0}(h)\right)
$$

Summing up, we have proved that

$$
\begin{aligned}
& \omega\left(\lambda^{*}\right) \in C^{2+\theta}\left(\Omega_{0}(h)\right) \cap W^{1,2}(\Omega) \cap L^{\infty}(\Omega) . \\
& \Delta \omega\left(\lambda^{*}\right)+\lambda^{*} m(x) \omega\left(\lambda^{*}\right)=0 \quad \text { in } \Omega_{0}(h) .
\end{aligned}
$$

Step 2: Next, we shall prove that the function $\omega\left(\lambda^{*}\right)$ is an eigenfunction of problem (1.7), more precisely, we shall show that, in some connected component $\Omega_{0}^{k}(h), 1 \leq k \leq N$, the function $\omega\left(\lambda^{*}\right)$ satisfies the conditions

$$
\begin{cases}-\Delta \omega\left(\lambda^{*}\right)=\lambda^{*} m(x) \omega\left(\lambda^{*}\right) & \text { in } \Omega_{0}^{k}(h), \\ \omega\left(\lambda^{*}\right)=0 & \text { on } \partial \Omega_{0}^{k}(h), \\ \omega\left(\lambda^{*}\right)>0 & \text { in } \Omega_{0}^{k}(h) .\end{cases}
$$

Step 2-a: First, by assertion (4.1) we remark that

$$
\lim _{\lambda_{n} \rightarrow \lambda^{*}}\left\|u\left(\lambda_{n}\right)\right\|_{L^{2}(\Omega)}=+\infty
$$

However, Lemma 3.2 tells us that, for each $\varepsilon>0$ there exists a constant $C\left(\varepsilon, \lambda^{*}\right)>$ 0 such that

$$
\left(u\left(\lambda_{n}\right)(x)\right)^{1-\varepsilon} \leq \frac{C\left(\varepsilon, \lambda^{*}\right)}{h^{*}(x)} \text { for all } x \in \Omega^{+}(h) .
$$

Hence it follows from assertion (5.7) and inequality (5.8) that

$$
\omega\left(\lambda^{*}\right)(x)=\lim _{\lambda_{n} \rightarrow \lambda^{*}} \omega\left(\lambda_{n}\right)(x)=\lim _{\lambda_{n} \rightarrow \lambda^{*}} \frac{u\left(\lambda_{n}\right)(x)}{\left\|u\left(\lambda_{n}\right)\right\|_{L^{2}(\Omega)}}=0
$$

for almost every $x \in \Omega^{+}(h)$. 
Therefore, combining assertions (5.5b) and (5.9) we find that

$$
\int_{\Omega_{0}(h)} \omega\left(\lambda^{*}\right)^{2} d x=\int_{\Omega} \omega\left(\lambda^{*}\right)^{2} d x=1 .
$$

This proves that the function $\omega\left(\lambda^{*}\right)$ is positive in some connected component $\Omega_{0}^{k}(h), 1 \leq k \leq N$, since we have

$$
\Omega_{0}(h)=\bigcup_{k=1}^{N} \Omega_{0}^{k}(h)
$$

Step 2-b: Furthermore, we can prove the following:

Lemma 5.2. The function $\omega\left(\lambda^{*}\right)$ satisfies the Dirichlet boundary condition

$$
\omega\left(\lambda^{*}\right)=0 \quad \text { on } \partial \Omega_{0}(h)=\bigcup_{k=1}^{N} \partial \Omega_{0}^{k}(h) .
$$

Step 2-c: Assuming Lemma 5.2 for the moment, we shall prove Proposition 5.1 .

By assertion (5.6) and Lemma 5.2, we find that the function $\omega\left(\lambda^{*}\right)$ is a positive eigenfunction of problem (1.7). This implies that $\lambda^{*}$ is the first eigenvalue of problem (1.7), so that

$$
\lambda^{*}=\lambda_{1}\left(\Omega_{0}^{k}(h)\right) \geq \mu_{1}\left(\Omega_{0}(h)\right) .
$$

The proof of Proposition 5.1 (and hence that of Theorem 1.2) is complete, apart from the proof of Lemma 5.2.

\section{Proof of Lemma 5.2.}

We show that the function $\omega\left(\lambda^{*}\right)$ satisfies the Dirichlet boundary condition

$$
\omega\left(\lambda^{*}\right)=0 \quad \text { on } \partial \Omega_{0}(h)
$$

or equivalently,

$$
\omega\left(\lambda^{*}\right) \in W_{0}^{1,2}\left(\Omega_{0}(h)\right)
$$

Step 1: First, we recall that

$$
\omega\left(\lambda^{*}\right) \in C^{2+\theta}\left(\Omega_{0}(h)\right) \cap W^{1,2}(\Omega) \cap L^{\infty}(\Omega),
$$


and that

$$
\omega\left(\lambda^{*}\right)=0 \quad \text { almost everywhere in } \Omega_{+}(h) .
$$

For $r>0$ sufficiently small, we let

$$
\Omega_{r}=\left\{x \in \Omega_{0}(h): \operatorname{dist}\left(x, \partial \Omega_{0}(h)\right)>r\right\},
$$

and let $\mathbf{n}$ be the unit exterior normal vector to the boundary $\partial \Omega_{r}$. One can construct a smooth vector function $\boldsymbol{\Psi}$ on $\Omega$ such that (see Figure 5.1)

$$
\Psi \cdot \mathbf{n} \geq \frac{1}{2} \quad \text { on } \partial \Omega_{r}
$$

and that

$$
\|\Psi\|_{C^{1}(\Omega)} \leq C
$$

with a constant $C>0$. Here and in the following the letter $C$ denotes a generic positive constant independent of $r$.

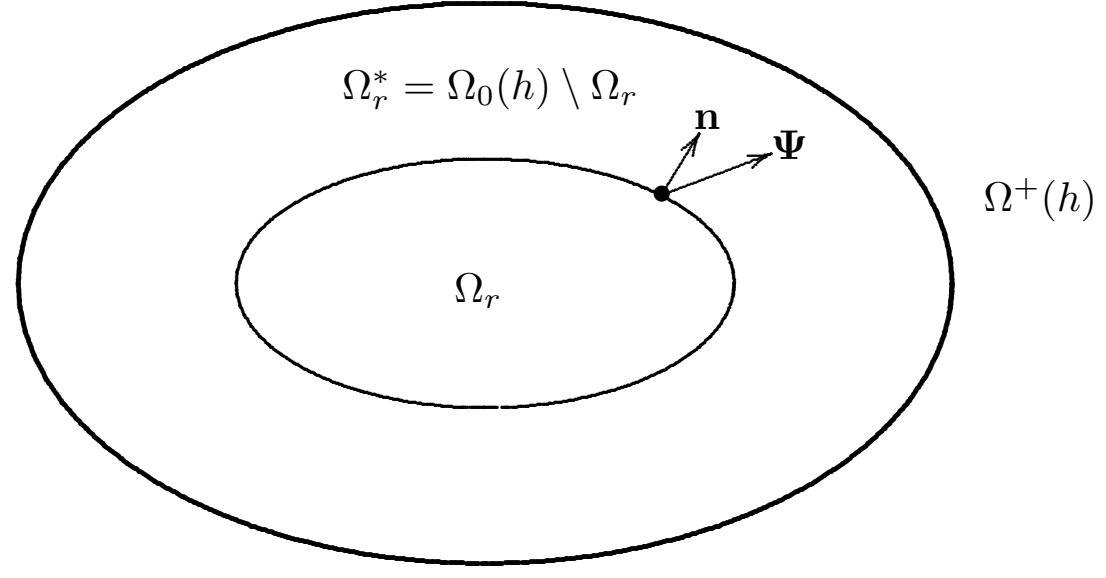

Figure 5.1

Since $\omega\left(\lambda^{*}\right)$ is in the Sobolev space $W_{0}^{1,2}(\Omega)$, we have, by Green's formula,

$$
\begin{aligned}
\int_{\Omega} \nabla \omega\left(\lambda^{*}\right) \cdot \Psi d x & =-\int_{\Omega} \omega\left(\lambda^{*}\right) \operatorname{div} \boldsymbol{\Psi} d x \\
& =-\int_{\Omega_{0}(h)} \omega\left(\lambda^{*}\right) \operatorname{div} \boldsymbol{\Psi} d x
\end{aligned}
$$


and also

$$
\begin{aligned}
\int_{\Omega} \nabla \omega\left(\lambda^{*}\right) \cdot \boldsymbol{\Psi} d x & =\int_{\Omega_{r}} \nabla \omega\left(\lambda^{*}\right) \cdot \boldsymbol{\Psi} d x+\int_{\Omega \backslash \Omega_{r}} \nabla \omega\left(\lambda^{*}\right) \cdot \boldsymbol{\Psi} d x \\
& =\int_{\Omega_{r}} \nabla \omega\left(\lambda^{*}\right) \cdot \boldsymbol{\Psi} d x+\int_{\Omega_{0}(h) \backslash \Omega_{r}} \nabla \omega\left(\lambda^{*}\right) \cdot \boldsymbol{\Psi} d x \\
& =\int_{\partial \Omega_{r}} \omega\left(\lambda^{*}\right)(\boldsymbol{\Psi} \cdot \mathbf{n}) d \sigma-\int_{\Omega_{r}} \omega\left(\lambda^{*}\right) \operatorname{div} \boldsymbol{\Psi} d x \\
& +\int_{\Omega_{0}(h) \backslash \Omega_{r}} \nabla \omega\left(\lambda^{*}\right) \cdot \boldsymbol{\Psi} d x .
\end{aligned}
$$

Thus, combining formulas (5.12) and (5.13) we obtain that

$$
\begin{aligned}
& \int_{\partial \Omega_{r}} \omega\left(\lambda^{*}\right)(\mathbf{\Psi} \cdot \mathbf{n}) d \sigma \\
= & -\int_{\Omega_{0}(h) \backslash \Omega_{r}} \omega\left(\lambda^{*}\right) \operatorname{div} \boldsymbol{\Psi} d x-\int_{\Omega_{0}(h) \backslash \Omega_{r}} \nabla \omega\left(\lambda^{*}\right) \cdot \boldsymbol{\Psi} d x .
\end{aligned}
$$

However, by using inequalities (5.11) and (5.4) we can estimate the first term on the right-hand side of formula (5.14) as follows:

$$
\left|\int_{\Omega_{0}(h) \backslash \Omega_{r}} \omega\left(\lambda^{*}\right) \operatorname{div} \boldsymbol{\Psi} d x\right| \leq C \int_{\Omega_{0}(h) \backslash \Omega_{r}} d x \leq C r .
$$

Furthermore, by using the Schwarz inequality and inequality (5.5c), we can estimate the second term on the right-hand side of formula (5.14) as follows:

$$
\begin{aligned}
& \left|\int_{\Omega_{0}(h) \backslash \Omega_{r}} \nabla \omega\left(\lambda^{*}\right) \cdot \boldsymbol{\Psi} d x\right| \\
\leq & C \int_{\Omega_{0}(h) \backslash \Omega_{r}}\left|\nabla \omega\left(\lambda^{*}\right)\right| d x \\
\leq & C\left(\int_{\Omega_{0}(h) \backslash \Omega_{r}}\left|\nabla \omega\left(\lambda^{*}\right)\right|^{2} d x\right)^{1 / 2}\left(\int_{\Omega_{0}(h) \backslash \Omega_{r}} d x\right)^{1 / 2} \\
\leq & C r^{\frac{1}{2}} .
\end{aligned}
$$

Hence, by formula (5.14), we have, for all $r>0$ sufficiently small,

$$
\int_{\partial \Omega_{r}} \omega\left(\lambda^{*}\right)(\mathbf{\Psi} \cdot \mathbf{n}) d \sigma \leq C r+C r^{\frac{1}{2}} \leq C r^{\frac{1}{2}}
$$


By inequality (5.10), this proves that, for all $r>0$ sufficiently small,

$$
\int_{\partial \Omega_{r}} \omega\left(\lambda^{*}\right) d \sigma \leq 2 \int_{\partial \Omega_{r}} \omega\left(\lambda^{*}\right)(\boldsymbol{\Psi} \cdot \mathbf{n}) d \sigma \leq C r^{\frac{1}{2}} .
$$

If we let

$$
\Omega_{r}^{*}=\Omega_{0}(h) \backslash \Omega_{r}
$$

then it follows from inequality (5.15) that

$$
\int_{\Omega_{r}^{*}} \omega\left(\lambda^{*}\right) d x=\int_{0}^{r}\left(\int_{\partial \Omega_{r}} \omega\left(\lambda^{*}\right) d \sigma\right) d t \leq C r^{\frac{3}{2}} .
$$

Therefore, we have, for all $r>0$ sufficiently small,

$$
\begin{aligned}
\left(\int_{\Omega_{r}^{*}} \omega\left(\lambda^{*}\right)^{2} d x\right)^{\frac{1}{2}} & =\left(\int_{\Omega_{r}^{*}} \omega\left(\lambda^{*}\right) \cdot \omega\left(\lambda^{*}\right) d x\right)^{\frac{1}{2}} \\
& \leq C\left(\int_{\Omega_{r}^{*}} \omega\left(\lambda^{*}\right) d x\right)^{\frac{1}{2}} \\
& \leq C r^{\frac{3}{4}}
\end{aligned}
$$

Step 2: Now we construct a sequence $\left\{\omega_{r}\right\}$ in the space $W^{1,2 n /(n+1)}\left(\Omega_{0}(h)\right)$ such that

$$
\omega_{r} \longrightarrow \omega\left(\lambda^{*}\right) \quad \text { in } W^{1, \frac{2 n}{n+1}}\left(\Omega_{0}(h)\right) \text { as } r \downarrow 0 \text {. }
$$

We let

$$
\begin{aligned}
& S_{r}=\left\{x \in \Omega: \operatorname{dist}\left(x, \partial \Omega_{0}(h)\right)<r\right\} \\
& S_{r}^{-}=\Omega_{0}(h) \backslash \overline{\Omega_{r}},
\end{aligned}
$$

and

$$
\begin{aligned}
& \partial S_{r}^{1}=\left\{x \in \Omega_{+}(h): \operatorname{dist}\left(x, \partial \Omega_{0}(h)\right)=r\right\}, \\
& \partial S_{r}^{2}=\left\{x \in \Omega_{0}(h): \operatorname{dist}\left(x, \partial \Omega_{0}(h)\right)=r\right\} .
\end{aligned}
$$

Then it is easy to see that, for all $r>0$ sufficiently small there exists a "shrinking" diffeomorphism

$$
\Psi: S_{r} \longrightarrow S_{r}^{-}
$$

with the following properties (see Figure 5.2):

$$
\Psi\left(\partial S_{r}^{1}\right)=\partial \Omega_{0}(h), \quad \Psi\left(\partial S_{r}^{2}\right)=\partial S_{r}^{2} .
$$




$$
\sup _{S_{r}}|\nabla \Psi| \leq C, \quad \sup _{S_{r}^{-}}\left|\nabla \Psi^{-1}\right| \leq C
$$

Indeed, in terms of local coordinates $\left(x_{1}, x_{2}, \cdots, x_{n-1}, x_{n}\right)$ such that

$$
\partial S_{r}^{1}=\left\{x_{n}=-r\right\}, \quad \partial S_{r}^{2}=\left\{x_{n}=+r\right\},
$$

the diffeomorphism $\Psi$ is given by the formula

$$
\Psi\left(x_{1}, x_{2}, \cdots, x_{n-1}, x_{n}\right)=\left(x_{1}, x_{2}, \cdots, x_{n-1}, \frac{x_{n}+r}{2}\right)
$$

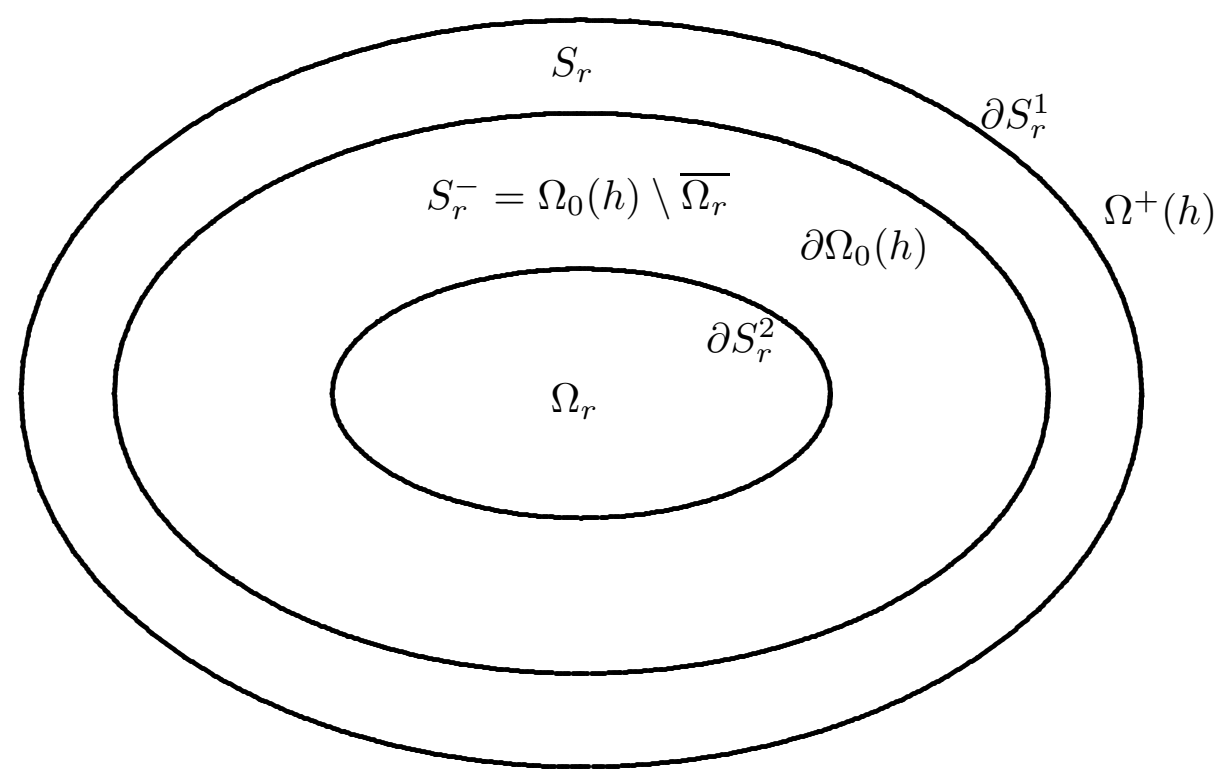

Figure 5.2

We let

$$
\omega_{r}(x)= \begin{cases}\omega\left(\lambda^{*}\right)\left(\Psi^{-1}(x)\right) & \text { if } x \in S_{r}^{-}, \\ \omega\left(\lambda^{*}\right)(x) & \text { if } x \in \Omega_{r} .\end{cases}
$$

Then, in view of assertion (5.9), it follows that the functions $\left\{\omega_{r}\right\}$ are in the space $W_{0}^{1,2}\left(\Omega_{0}(h)\right)$ for all $r>0$ sufficiently small. Next, by inequality (5.16) we have, for all $r>0$ sufficiently small,

$$
\begin{aligned}
\left\|\omega_{r}-\omega\left(\lambda^{*}\right)\right\|_{L^{2}\left(\Omega_{0}(h)\right)} & =\left\|\omega_{r}-\omega\left(\lambda^{*}\right)\right\|_{L^{2}\left(\Omega_{0}(h) \backslash \Omega_{r}\right)} \\
& \leq\left\|\omega_{r}\right\|_{L^{2}\left(\Omega_{0}(h) \backslash \Omega_{r}\right)}+\left\|\omega\left(\lambda^{*}\right)\right\|_{L^{2}\left(\Omega_{0}(h) \backslash \Omega_{r}\right)} \\
& \leq 2\left\|\omega\left(\lambda^{*}\right)\right\|_{L^{2}\left(\Omega_{r}^{*}\right)} \\
& \leq C r^{\frac{3}{4}} .
\end{aligned}
$$


Furthermore, using Hölder's inequality we obtain that, for all $r>0$ sufficiently small,

$$
\begin{aligned}
\left\|\omega_{r}-\omega\left(\lambda^{*}\right)\right\|_{L^{2 n /(n+1)}\left(\Omega_{0}(h)\right)} & \leq\left|\Omega_{0}(h)\right|^{\frac{n+1}{2 n}-\frac{1}{2}}\left\|\omega_{r}-\omega\left(\lambda^{*}\right)\right\|_{L^{2}\left(\Omega_{r}^{*}\right)} \\
& \leq C r^{\frac{3}{4}} .
\end{aligned}
$$

Similarly, it follows that, for all $r>0$ sufficiently small,

$$
\begin{aligned}
& \left\|\nabla\left(\omega_{r}-\omega\left(\lambda^{*}\right)\right)\right\|_{L^{2 n /(n+1)}\left(\Omega_{0}(h)\right)} \\
= & \| \nabla\left(\omega_{r}-\omega\left(\lambda^{*}\right) \|_{L^{2 n /(n+1)}\left(\Omega_{0}(h) \backslash \Omega_{r}\right)}\right. \\
\leq & \left\|\nabla \omega_{r}\right\|_{L^{2 n /(n+1)}\left(\Omega_{r}^{*}\right)}+\left\|\nabla \omega\left(\lambda^{*}\right)\right\|_{L^{2 n /(n+1)}\left(\Omega_{r}^{*}\right)} \\
\leq & \sup \left|\nabla \Psi^{-1}\right|\left\|\nabla \omega\left(\lambda^{*}\right)\right\|_{L^{2 n /(n+1)}\left(\Omega_{r}^{*}\right)}+\left\|\nabla \omega\left(\lambda^{*}\right)\right\|_{L^{2 n /(n+1)}\left(\Omega_{r}^{*}\right)} \\
\leq & C\left\|\nabla \omega\left(\lambda^{*}\right)\right\|_{L^{2 n /(n+1)}\left(\Omega_{r}^{*}\right)} .
\end{aligned}
$$

However, we find that the last term can be estimated as follows:

$$
\begin{aligned}
\left\|\nabla \omega\left(\lambda^{*}\right)\right\|_{L^{2 n /(n+1)\left(\Omega_{r}^{*}\right)}} & =\left(\int_{\Omega_{r}^{*}}\left|\nabla \omega\left(\lambda^{*}\right)\right|^{\frac{2 n}{n+1}} d x\right)^{\frac{n+1}{2 n}} \\
& \leq\left(\left|\Omega_{r}^{*}\right|^{1-\frac{n}{n+1}}\left(\int_{\Omega_{r}^{*}}\left|\nabla \omega\left(\lambda^{*}\right)\right|^{2} d x\right)^{\frac{n+1}{n}}\right)^{\frac{n+1}{2 n}} \\
& \leq\left|\Omega_{r}^{*}\right|^{\frac{1}{2 n}}\left\|\nabla \omega\left(\lambda^{*}\right)\right\|_{L^{2}(\Omega)} \\
& \leq C r^{\frac{1}{2 n}}
\end{aligned}
$$

Hence we obtain that, for all $r>0$ sufficiently small,

$$
\left\|\nabla\left(\omega_{r}-\omega\left(\lambda^{*}\right)\right)\right\|_{L^{2 n /(n+1)}\left(\Omega_{0}(h)\right)} \leq C r^{\frac{1}{2 n}}
$$

Therefore, combining inequalities (5.18) and (5.19) we have proved that

$$
\omega\left(\lambda^{*}\right) \in W_{0}^{1, \frac{2 n}{n+1}}\left(\Omega_{0}(h)\right),
$$

and that

$$
\omega_{r} \longrightarrow \omega\left(\lambda^{*}\right) \quad \text { in } W_{0}^{1, \frac{2 n}{n+1}}\left(\Omega_{0}(h)\right) \text { as } r \downarrow 0 \text {. }
$$

Step 3: Finally, we show that

$$
\omega\left(\lambda^{*}\right) \in W_{0}^{1,2}\left(\Omega_{0}(h)\right),
$$


and that

$$
\omega_{r} \longrightarrow \omega\left(\lambda^{*}\right) \quad \text { in } W_{0}^{1,2}\left(\Omega_{0}(h)\right) \text { as } r \downarrow 0 \text {. }
$$

We recall that the function

$$
\omega\left(\lambda^{*}\right) \in W_{0}^{1, \frac{2 n}{n+1}}\left(\Omega_{0}(h)\right) \cap L^{\infty}\left(\Omega_{0}(h)\right)
$$

satisfies the equation

$$
\Delta \omega\left(\lambda^{*}\right)+\lambda^{*} m(x) \omega\left(\lambda^{*}\right)=0 \quad \text { in } \Omega_{0}(h) .
$$

Thus, by using $L^{p}$ estimates for elliptic equations (cf. Gilbarg-Trudinger [11, Theorem 9.14]) we obtain that

$$
\omega\left(\lambda^{*}\right) \in W^{2, \frac{2 n}{n+1}}\left(\Omega_{0}(h)\right) \cap W_{0}^{1, \frac{2 n}{n+1}}\left(\Omega_{0}(h)\right) .
$$

On the other hand, by applying the Sobolev imbedding theorem (cf. AdamsFournier [1, Theorem 4.12]) we find that the injection

$$
W^{2, \frac{2 n}{n+1}}\left(\Omega_{0}(h)\right) \subset W^{1, \frac{2 n}{n-1}}\left(\Omega_{0}(h)\right)
$$

is continuous.

Hence it follows from assertions (5.21) and (5.22) that

$$
\left\|\nabla \omega\left(\lambda^{*}\right)\right\|_{L^{2 n /(n-1)}\left(\Omega_{0}(h)\right)} \leq C .
$$

By virtue of Hölder's inequality, this proves that, for all $r>0$ sufficiently small,

$$
\begin{aligned}
\left(\int_{\Omega_{r}^{*}}\left|\nabla \omega\left(\lambda^{*}\right)\right|^{2} d x\right)^{\frac{1}{2}} & \leq\left(\int_{\Omega_{r}^{*}}\left|\nabla \omega\left(\lambda^{*}\right)\right|^{\frac{2 n}{n-1}} d x\right)^{\frac{n-1}{2 n}}\left(\int_{\Omega_{r}^{*}} d x\right)^{\frac{1}{2 n}} \\
& =\left\|\nabla \omega\left(\lambda^{*}\right)\right\|_{L^{2 n /(n-1)}\left(\Omega_{0}(h)\right)}\left|\Omega_{r}^{*}\right|^{\frac{1}{2 n}} \\
& \leq C r^{\frac{1}{2 n}}
\end{aligned}
$$

Thus we have, for all $r>0$ sufficiently small,

$$
\begin{aligned}
\left\|\nabla\left(\omega_{r}-\omega\left(\lambda^{*}\right)\right)\right\|_{L^{2}\left(\Omega_{0}(h)\right)} & =\left\|\nabla\left(\omega_{r}-\omega\left(\lambda^{*}\right)\right)\right\|_{L^{2}\left(\Omega_{r}^{*}\right)} \\
& \leq C\left\|\nabla \omega\left(\lambda^{*}\right)\right\|_{L^{2}\left(\Omega_{r}^{*}\right)} \\
& \leq C r^{\frac{1}{2 n}}
\end{aligned}
$$

Therefore, assertion (5.20) follows by combining inequalities (5.17) and (5.23). 
The proof of Lemma 5.2 and hence that of Proposition 5.1 is complete.

\section{Proof of Theorem 1.3}

In this section we prove Theorem 1.3 by using comparison theorems based on the maximum principle just as in Fraile et al. [9, Theorem 3.7], Pao [18, Chapter 5, Theorem 4.4] and Sattinger [22, Theorem 2.6.2].

Step 1: First, we consider the case where $0<\lambda<\lambda_{1}(m)$ : In this case, we know from Hess [12, Chapter II, Section 15] that there exist a constant $\sigma_{1}(\lambda) \geq 0$ and a function $\varphi_{1}(x) \in C^{2+\theta}(\bar{\Omega})$ such that

$$
\begin{cases}(-\Delta-\lambda m(x)) \varphi_{1}=\sigma_{1}(\lambda) \varphi_{1} & \text { in } \Omega \\ \varphi_{1}>0 & \text { in } \Omega \\ \varphi_{1}=0 & \text { on } \partial \Omega\end{cases}
$$

Moreover, it is easy to verify that the functions $\kappa \varphi_{1}(x), \kappa>0$, are supersolutions of problem (1.6). Indeed, we have

$$
\begin{cases}(-\Delta-\lambda m(x))\left(\kappa \varphi_{1}\right) \geq 0 & \text { in } \Omega, \\ \kappa \varphi_{1}=0 & \text { on } \partial \Omega .\end{cases}
$$

Hence, if $w\left(x, t ; \kappa \varphi_{1}\right)$ is a unique classical global solution of problem (1.11) with initial value $\kappa \varphi_{1}$

$$
\begin{cases}\frac{\partial w}{\partial t}=\frac{1}{\lambda} \Delta w+(m(x)-h(x) w) w & \text { in } \Omega \times(0, \infty), \\ w=0 & \text { on } \partial \Omega \times(0, \infty), \\ \left.w\right|_{t=0}=\kappa \varphi_{1} & \text { in } \Omega,\end{cases}
$$

then it follows from an application of Pao [18, Chapter 5, Theorem 4.3] and Sattinger [22, Theorem 2.6.1]) that

$$
\lim _{t \rightarrow \infty}\left\|w\left(\cdot, t ; \kappa \varphi_{1}\right)\right\|_{C(\bar{\Omega})}=0
$$

since the zero solution is a unique steady state solution of problem (1.11) for $0<\lambda<\lambda_{1}(m)$.

Now let $u_{0}$ be an arbitrary function in $C^{2+\theta}(\bar{\Omega})$ satisfying the conditions

$$
\begin{cases}u_{0} \geq 0 & \text { in } \Omega, \\ u_{0}=0 & \text { on } \partial \Omega .\end{cases}
$$


Then we can find a constant $\kappa>0$ such that

$$
0 \leq u_{0}(x) \leq \kappa \varphi_{1}(x) \text { in } \Omega
$$

If $w\left(x, t ; u_{0}\right)$ and $w\left(x, t ; \kappa \varphi_{1}\right)$ are solutions of problem (1.11) with initial values $u_{0}$ and $\kappa \varphi_{1}$ respectively, then, applying the comparison theorem for problem (1.11) (see Pao [18, Chapter 5, Theorem 4.3], Sattinger [22, Theorem 2.5.2]) we obtain that

$$
0 \leq w\left(x, t ; u_{0}\right) \leq w\left(x, t ; \kappa \varphi_{1}\right) \quad \text { in } \Omega \times[0, \infty) .
$$

Therefore, we have, by assertions (6.1) and (6.2),

$$
\lim _{t \rightarrow \infty}\left\|w\left(\cdot, t ; u_{0}\right)\right\|_{C(\bar{\Omega})}=0 .
$$

This proves that the zero solution of problem (1.6) is globally asymptotically stable for $0<\lambda<\lambda_{1}(m)$.

It remains to prove the decay estimate (1.13)

$$
\begin{aligned}
& \int_{\Omega} w\left(x, t ; u_{0}\right) d x \\
\leq & \exp \left[-\left(\frac{1}{\lambda}-\frac{1}{\lambda_{1}(m)}\right) \lambda_{1}(1) t\right]|\Omega|^{1 / 2}\left(\int_{\Omega} u_{0}(x)^{2} d x\right)^{1 / 2}, \quad t>0 .
\end{aligned}
$$

We let

$$
E(t)=\frac{1}{2} \int_{\Omega} w\left(x, t ; u_{0}\right)^{2} d x
$$

Then, by integration by parts it follows that

$$
\begin{aligned}
E^{\prime}(t) & =\int_{\Omega} w \cdot w_{t} d x \\
& =\frac{1}{\lambda} \int_{\Omega} \Delta w \cdot w d x+\int_{\Omega} m(x) w^{2} d x-\int_{\Omega} h(x) w^{3} d x \\
& =-\frac{1}{\lambda} \int_{\Omega}|\nabla w|^{2} d x+\int_{\Omega} m(x) w^{2} d x-\int_{\Omega} h(x) w^{3} d x \\
& \leq-\frac{1}{\lambda} \int_{\Omega}|\nabla w|^{2} d x+\frac{1}{\lambda_{1}(m)} \int_{\Omega}|\nabla w|^{2} d x .
\end{aligned}
$$

However, we have

$$
\int_{\Omega}|\nabla w|^{2} d x \geq \lambda_{1}(1) \int_{\Omega} w^{2} d x
$$

and so

$$
-\left(\frac{1}{\lambda}-\frac{1}{\lambda_{1}(m)}\right) \int_{\Omega}|\nabla u|^{2} d x \leq-\left(\frac{1}{\lambda}-\frac{1}{\lambda_{1}(m)}\right) \lambda_{1}(1) \int_{\Omega} w^{2} d x .
$$


Therefore, carrying inequality (6.4) into inequality (6.3) we obtain that

$$
\begin{aligned}
E^{\prime}(t) & \leq-\left(\frac{1}{\lambda}-\frac{1}{\lambda_{1}(m)}\right) \lambda_{1}(1) \int_{\Omega} w^{2} d x \\
& =-2\left(\frac{1}{\lambda}-\frac{1}{\lambda_{1}(m)}\right) \lambda_{1}(1) E(t)
\end{aligned}
$$

This implies that

$$
\begin{aligned}
E(t) & \leq \exp \left[-2\left(\frac{1}{\lambda}-\frac{1}{\lambda_{1}(m)}\right) \lambda_{1}(1) t\right] E(0) \\
& =\frac{1}{2} \exp \left[-2\left(\frac{1}{\lambda}-\frac{1}{\lambda_{1}(m)}\right) \lambda_{1}(1) t\right] \int_{\Omega} u_{0}(x)^{2} d x .
\end{aligned}
$$

On the other hand, we have, by Schwarz's inequality,

$$
\int_{\Omega} w d x \leq\left(\int_{\Omega} w^{2} d x\right)^{1 / 2}\left(\int_{\Omega} 1^{2} d x\right)^{1 / 2}=\sqrt{2}|\Omega|^{1 / 2} E(t)^{1 / 2} .
$$

The desired inequality (1.13) follows by combining inequalities (6.6) and (6.5).

Step 2: Secondly, we consider the case where $\lambda_{1}(m)<\lambda<\mu_{1}\left(\Omega_{0}(h)\right)$ : Let $v \in C^{2+\theta}(\bar{\Omega})$ be a unique solution of the Dirichlet problem

$$
\begin{cases}-\Delta v=1 & \text { in } \Omega \\ v=0 & \text { on } \partial \Omega\end{cases}
$$

Note that

$$
v>0 \text { in } \Omega
$$

and that

$$
\min _{x \in K} h(x) v(x)>0
$$

for any compact set $K \subset \Omega \backslash \Omega_{0}(h)$.

Then, by applying Fraile et al. [9, Theorem 2.4] to our situation we have the following:

Claim 6.1. For a positive integer $k$ sufficiently large, we can find a constant $\sigma_{k}(\lambda)>0$ and a function $\varphi \in C^{2+\theta}(\bar{\Omega})$ such that

$$
\begin{cases}(-\Delta-\lambda m(x)+\lambda k h(x) v(x)) \varphi=\sigma_{k}(\lambda) \varphi & \text { in } \Omega \\ \varphi>0 & \text { in } \Omega \\ \varphi=0 & \text { on } \partial \Omega .\end{cases}
$$


If we take a constant $\kappa>0$ so large that

$$
\kappa \varphi(x) \geq k v(x) \quad \text { in } \Omega
$$

then it follows that the function $\kappa \varphi(x)$ is a supersolution of problem (1.6). Indeed, we have, by assertion (6.7),

$$
\begin{aligned}
& (-\Delta-\lambda m(x))(\kappa \varphi)+\lambda h(x)(\kappa \varphi)^{2} \\
= & (-\Delta-\lambda m(x)+\lambda k h(x) v(x))(\kappa \varphi)+\lambda h(x)(\kappa \varphi)^{2}-\lambda k h(x) v(x)(\kappa \varphi) \\
= & \kappa \sigma_{k}(\lambda) \varphi(x)+\lambda \kappa h(x)(\kappa \varphi(x)-k v(x)) \varphi(x) \\
\geq & 0 \quad \text { in } \Omega,
\end{aligned}
$$

and also

$$
\kappa \varphi=0 \quad \text { on } \partial \Omega
$$

On the other hand, we can find a constant $\mu_{1}(\lambda)<0$ and a function $\varphi_{1} \in$ $C^{2+\theta}(\bar{\Omega})$ such that

$$
\begin{cases}(-\Delta-\lambda m(x)) \varphi_{1}=\mu_{1}(\lambda) \varphi_{1} & \text { in } \Omega, \\ \varphi_{1}>0 & \text { in } \Omega, \\ \varphi_{1}=0 & \text { on } \partial \Omega .\end{cases}
$$

Then it follows (cf. Fraile et al. [9, Lemma 3.3]) that $\varepsilon \varphi_{1}(x)$ is a subsolution of problem (1.6) if $\varepsilon$ is so small that

$$
\mu_{1}(\lambda)+\lambda \varepsilon h(x) \varphi_{1}(x) \leq 0 \quad \text { in } \Omega
$$

Indeed, we have

$$
\begin{aligned}
(-\Delta-\lambda m(x))\left(\varepsilon \varphi_{1}\right)+\lambda h(x)\left(\varepsilon \varphi_{1}\right)^{2} & =\varepsilon\left(\mu_{1}(\lambda)+\lambda \varepsilon h(x) \varphi_{1}(x)\right) \varphi_{1}(x) \\
& \leq 0 \text { in } \Omega
\end{aligned}
$$

and also

$$
\varepsilon \varphi_{1}=0 \quad \text { on } \partial \Omega
$$

Now let $u_{0}$ be an arbitrary nontrivial function in $C^{2+\theta}(\bar{\Omega})$ satisfying conditions (1.12). Then we can find constants $\varepsilon>0$ and $\kappa>0$ such that

$$
\varepsilon \varphi_{1}(x) \leq u_{0}(x) \leq \kappa \varphi(x) \quad \text { in } \Omega
$$


If $w\left(x, t ; u_{0}\right)$ is a unique classical global solution of problem (1.11), then, applying Pao [18, Chapter 5, Theorem 4.4] and Sattinger [22, Theorem 2.6.2] we obtain that

$$
\max _{x \in \bar{\Omega}}\left|w\left(x, t ; u_{0}\right)-u(\lambda)(x)\right| \longrightarrow 0 \quad \text { as } t \rightarrow \infty .
$$

This proves that the positive solution $u(\lambda)$ of problem (1.6) is globally asymptotically stable for each $\lambda \in\left(\lambda_{1}(m), \mu_{1}\left(\Omega_{0}(h)\right)\right.$.

Step 3: Finally, we consider the case where $\lambda>\mu_{1}\left(\Omega_{0}(h)\right)$.

Assume, to the contrary, that there exists a nontrivial initial value $u_{0} \in C^{2+\theta}(\bar{\Omega})$ satisfying conditions (1.12) such that

$$
\sup _{t \geq 0}\left\|w\left(\cdot, t ; u_{0}\right)\right\|_{C(\bar{\Omega})}<\infty .
$$

Then, by Redlinger [20, Satz] it follows that

$$
\sup _{t \geq 0}\left\|w\left(\cdot, t ; u_{0}\right)\right\|_{C^{2}(\bar{\Omega})}<\infty .
$$

Hence we can find a function $v_{0} \in C^{2+\theta}(\bar{\Omega})$ satisfying conditions (1.12) such that

$$
0 \leq w\left(x, t ; u_{0}\right) \leq v_{0}(x) \text { in } \Omega \times(0, \infty)
$$

Furthermore, there exist a constant $\mu_{0}(\lambda)<0$ and a function $\psi \in C^{2+\theta}(\bar{\Omega})$ such that

$$
\begin{cases}\left(-\Delta-\lambda m(x)+\lambda h(x) v_{0}(x)\right) \psi=\mu_{0}(\lambda) \psi & \text { in } \Omega, \\ \psi>0 & \text { in } \Omega, \\ \psi=0 & \text { on } \partial \Omega .\end{cases}
$$

Then we consider the following linear parabolic initial boundary value problem:

$$
\begin{cases}\frac{\partial z}{\partial t}=\frac{1}{\lambda} \Delta z+\left(m(x)-h(x) v_{0}(x)\right) z & \text { in } \Omega \times(0, \infty) \\ z=0 & \text { on } \partial \Omega \times(0, \infty) \\ \left.z\right|_{t=0}=u_{0} & \text { in } \Omega\end{cases}
$$

If $z\left(x, t ; u_{0}\right)$ is a unique classical global solution of problem $(6.10)$, then, arguing as in the proof of Pao [18, Chapter 5, Theorem 3.3] we obtain that

$$
\liminf _{t \rightarrow \infty} \max _{x \in \bar{\Omega}} z\left(x, t ; u_{0}\right) \geq \rho \max _{x \in \bar{\Omega}} \psi(x)
$$

for any $\rho>0$. In particular, this implies that

$$
\lim _{t \rightarrow \infty}\left\|z\left(\cdot, t ; u_{0}\right)\right\|_{C(\bar{\Omega})}=\infty
$$


On the other hand, by condition (6.9) it follows that

$$
\begin{aligned}
& \left(\frac{\partial w}{\partial t}-\frac{1}{\lambda} \Delta\right) w-\left(m(x)-h(x) v_{0}(x)\right) w \\
= & (m(x)-h(x) w) w-\left(m(x)-h(x) v_{0}(x)\right) w \\
= & h(x)\left(v_{0}(x)-w\left(x, t ; u_{0}\right)\right) w\left(x, t ; u_{0}\right) \\
\geq & 0 \\
= & \left(\frac{\partial z}{\partial t}-\frac{1}{\lambda} \Delta\right) z-\left(m(x)-h(x) v_{0}(x)\right) z \text { in } \Omega \times(0, \infty),
\end{aligned}
$$

and that

$$
\begin{aligned}
& w=0=z \quad \text { on } \partial \Omega \times(0, \infty), \\
& \left.w\right|_{t=0}=u_{0}=\left.z\right|_{t=0} \quad \text { in } \Omega .
\end{aligned}
$$

Therefore, applying the comparison theorem for problem (6.10) (see Pao [18, Chapter 5, Theorem 4.3], Sattinger [22, Theorem 2.5.2]) we obtain that

$$
w\left(x, t ; u_{0}\right) \geq z\left(x, t ; u_{0}\right) \geq 0 \quad \text { in } \Omega \times(0, \infty)
$$

Hence we have, by assertions (6.11) and (6.12),

$$
\lim _{t \rightarrow \infty}\left\|w\left(\cdot, t ; u_{0}\right)\right\|_{C(\bar{\Omega})}=\infty
$$

This contradicts hypothesis (6.8).

The proof of Theorem 1.3 is now complete.

\section{The Neumann Case}

In this final section we study problem (1.16) with homogeneous Neumann condition. The same general approach to problem (1.1) with homogeneous Dirichlet condition can still be used, although the analysis may be somewhat different, since the operator $-\Delta$ will have zero as an eigenvalue.

The next theorem (see Brown-Lin [4, Theorem 3.13], Senn-Hess [24, Theorems 2 and 3]) asserts the existence of the first eigenvalue of the linearized Neumann problem with indefinite weight function $m(x)$ and positive parameter $\lambda=1 / d$ :

$$
\begin{cases}-\Delta \phi=\lambda m(x) \phi & \text { in } \Omega, \\ \frac{\partial \phi}{\partial \mathbf{n}}=0 & \text { on } \partial \Omega .\end{cases}
$$


Theorem 7.1. Assume that the function $m(x) \in C^{\theta}(\bar{\Omega})$ attains both positive and negative values in $\Omega$. Then problem (7.1) admits a unique nonnegative eigenvalue $\mu_{1}(m)$ having a positive eigenfunction, and we have

$$
\begin{cases}\mu_{1}(m)>0 & \text { if } \int_{\Omega} m(x) d x<0 \\ \mu_{1}(m)=0 & \text { if } \int_{\Omega} m(x) d x \geq 0\end{cases}
$$

Next we consider the steady state problem (1.16) with $d=1 / \lambda$ :

$$
\begin{cases}-\Delta u=\lambda(m(x)-h(x) u) u & \text { in } \Omega \\ \frac{\partial u}{\partial \mathbf{n}}=0 & \text { on } \partial \Omega .\end{cases}
$$

Then we have the following generalization of Hess [12, Theorem 27.1] to the case where $h(x)$ may vanish in $\Omega$ (cf. Fraile et al. [9, Theorem 3.7], Senn [23, Theorem $3.2])$ :

Theorem 7.2. Assume that $h(x) \in C^{1}(\bar{\Omega})$ satisfies condition $(Z)$ and that each set $\left\{x \in \Omega_{0}^{i}(h): m(x)>0\right\}, 1 \leq i \leq N$, has positive measure. Then problem (7.2) has a unique positive solution $u(\lambda) \in C^{2+\theta}(\bar{\Omega})$ for every $\lambda \in\left(\mu_{1}(m), \mu_{1}\left(\Omega_{0}(h)\right)\right)$. For any $\lambda \geq \mu_{1}\left(\Omega_{0}(h)\right)$, there exists no positive solution of problem (7.2). Moreover, we have (see Figures 7.1, 7.2 and 7.3)

$$
\lim _{\lambda \rightarrow \mu_{1}\left(\Omega_{0}(h)\right)}\|u(\lambda)\|_{L^{2}(\Omega)}=+\infty
$$

and also

$$
\lim _{\lambda \rightarrow \mu_{1}(m)}\|u(\lambda)-c\|_{C^{2+\theta}(\bar{\Omega})}=0
$$

where

$$
c=\max \left\{\frac{\int_{\Omega} m(x) d x}{\int_{\Omega} h(x) d x}, 0\right\} .
$$

Remark 7.1. Assume that $h(x)>0$ on $\bar{\Omega}$, and that the function $m(x)$ attains both positive and negative values in $\Omega$ and $\int_{\Omega} m(x) d x<0$. Then, arguing as in the proof of Cantrell-Cosner [5, Theorem 4.1] and using Brown-Lin [4, Theorem 3.13] we can give an estimate of the decay rate of the total size of the positive steady states $u(\lambda)$ as $\lambda \downarrow \mu_{1}(m)$ :

$$
\int_{\Omega} u(\lambda) d x \leq\left(1-\frac{\mu_{1}(m)}{\lambda}\right)|\Omega|^{2 / 3} \frac{\left(\int_{\Omega}\left(m^{+}\right)^{3} d x\right)^{1 / 3}}{\min _{x \in \bar{\Omega}} h(x)}, \quad \lambda>\mu_{1}(m) .
$$


Theorem 7.3. (i) Assume that

$$
\int_{\Omega} m(x) d x<0 .
$$

Then the zero solution of problem (7.2) is globally asymptotically stable if $\lambda$ is so small that

$$
0<\lambda<\mu_{1}(m) .
$$

In this case we can give an estimate of the decay rate of the total size of the population as $t \downarrow 0$ :

$$
\begin{aligned}
& \int_{\Omega} w\left(x, t ; u_{0}\right) d x \\
\leq & \exp \left[-\left(\frac{1}{\lambda}-\frac{1}{\mu_{1}(m)}\right) \mu_{1}(1) t\right]|\Omega|^{1 / 2}\left(\int_{\Omega} u_{0}(x)^{2} d x\right)^{1 / 2}, \quad t>0 .
\end{aligned}
$$

(ii) A positive solution $u(\lambda)$ of problem (7.2) is globally asymptotically stable for each $\lambda$ satisfying the condition

$$
\mu_{1}(m)<\lambda<\mu_{1}\left(\Omega_{0}(h)\right) .
$$

(iii) If $\lambda$ is so large that

$$
\lambda>\mu_{1}\left(\Omega_{0}(h)\right),
$$

then we have

$$
\max _{x \in \bar{\Omega}}\left|w\left(x, t ; u_{0}\right)\right| \longrightarrow \infty \quad \text { as } t \rightarrow \infty
$$

for each nontrivial $u_{0} \in C^{2+\theta}(\bar{\Omega})$ satisfying conditions (1.12).

The proofs of Theorems 7.2 and 7.3 are essentially the same as those of Theorems 1.2 and 1.3 , respectively.

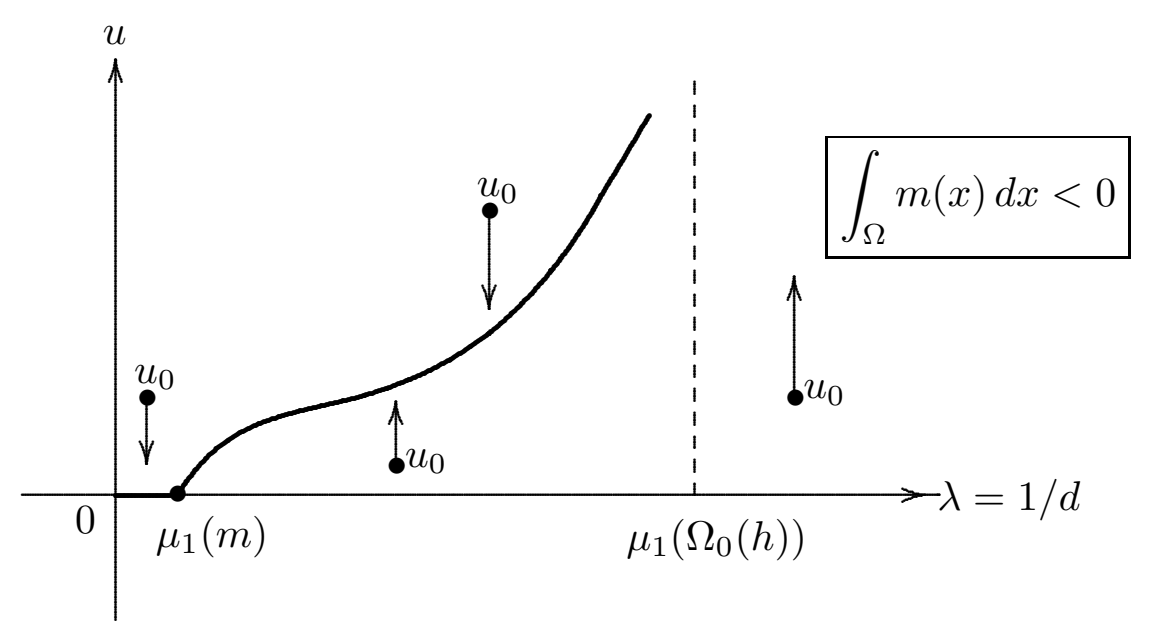

Figure 7.1 


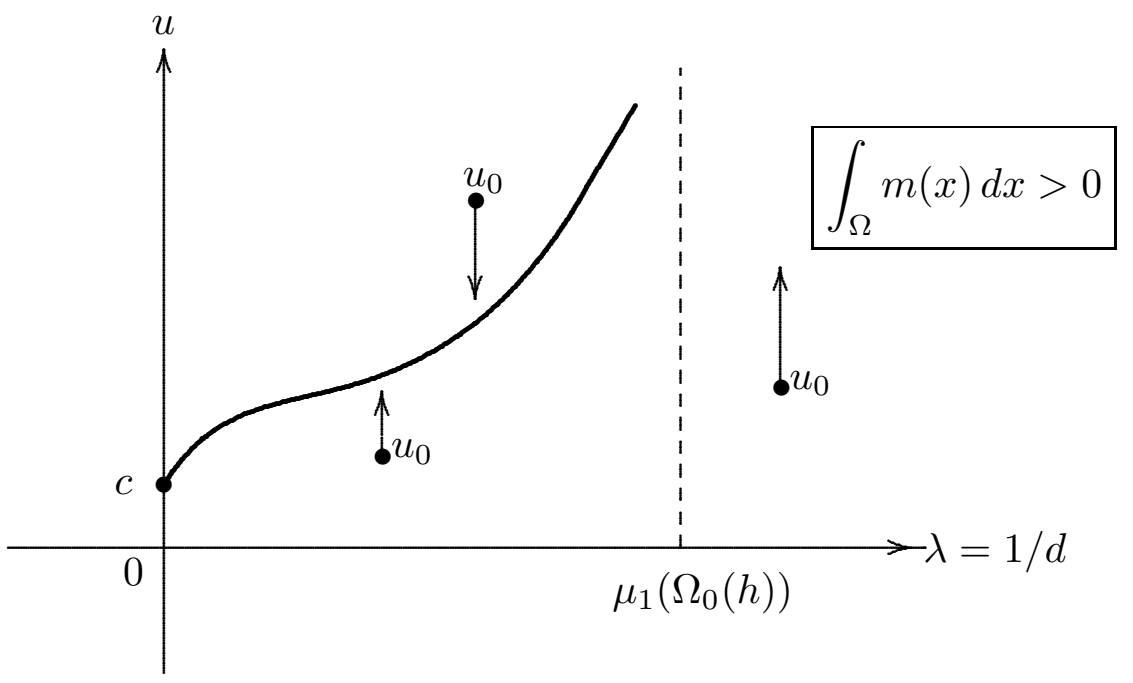

Figure 7.2

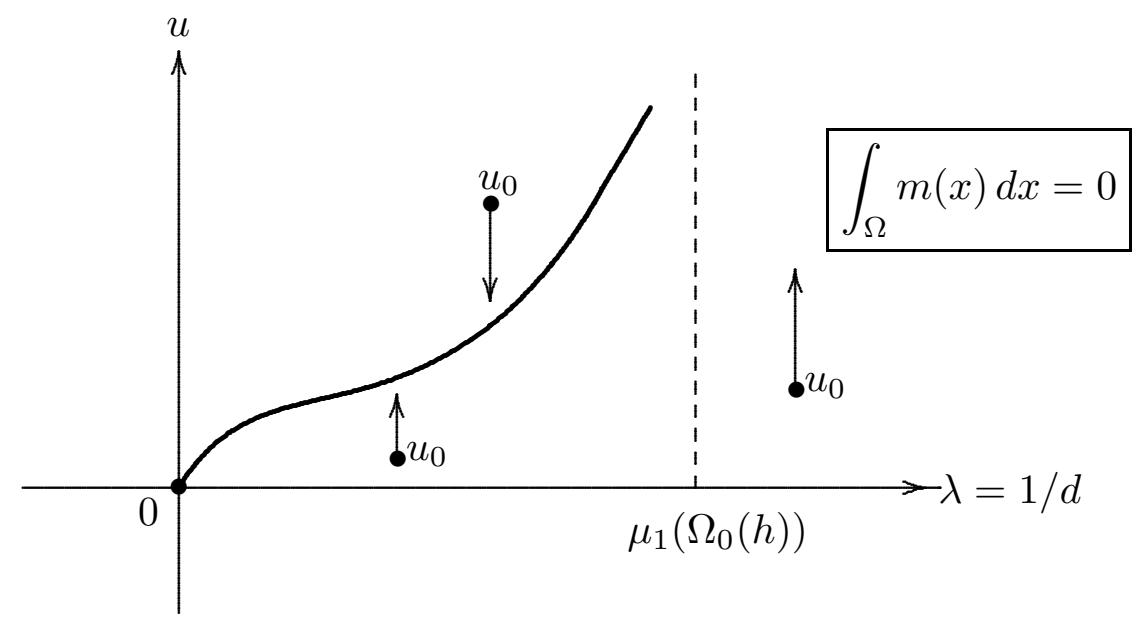

Figure 7.3

\section{Appendix: Proof of Remark 1.3}

This appendix is devoted to the proof of Remark 1.3. Namely, we prove that if $h(x)>0$ on $\bar{\Omega}$ and if $m(x)$ attains positive values in $\Omega$, then the decay estimate (1.10) holds true as $\lambda \downarrow \lambda_{1}(m)$ :

$$
\int_{\Omega} u(\lambda) d x \leq\left(1-\frac{\lambda_{1}(m)}{\lambda}\right)|\Omega|^{2 / 3} \frac{\left(\int_{\Omega}\left(m^{+}\right)^{3} d x\right)^{1 / 3}}{\min _{x \in \bar{\Omega}} h(x)}, \quad \lambda>\lambda_{1}(m) .
$$


Our proof here is inspired by the proof of Cantrell-Cosner [5, Theorem 4.1].

Let $u(\lambda) \in C^{2}(\bar{\Omega})$ be a positive solution of problem (1.6) for $\lambda_{1}(m)<\lambda<$ $\mu_{1}\left(\Omega_{0}(h)\right)$. In the proof of Lemma 4.1 , we have proved the formula

$$
\frac{1}{\lambda} \int_{\Omega}|\nabla u(\lambda)|^{2} d x+\int_{\Omega} h(x) u(\lambda)^{3} d x=\int_{\Omega} m(x) u(\lambda)^{2} d x .
$$

This implies that

$$
\int_{\Omega} m(x) u(\lambda)^{2} d x>0
$$

Hence we have, by the variational formula (1.4),

$$
\lambda_{1}(m) \int_{\Omega} m(x) u(\lambda)^{2} d x \leq \int_{\Omega}|\nabla u(\lambda)|^{2} d x .
$$

By formula (A.1) and inequality (A.2), it follows that

$$
\begin{aligned}
\int_{\Omega} h(x) u(\lambda)^{3} d x & =\int_{\Omega} m(x) u(\lambda)^{2} d x-\frac{1}{\lambda} \int_{\Omega}|\nabla u(\lambda)|^{2} d x \\
& \leq \int_{\Omega} m(x) u(\lambda)^{2} d x-\frac{\lambda_{1}(m)}{\lambda} \int_{\Omega} m(x) u(\lambda)^{2} d x \\
& =\left(1-\frac{\lambda_{1}(m)}{\lambda}\right) \int_{\Omega} m(x) u(\lambda)^{2} d x .
\end{aligned}
$$

Furthermore, we have, by Hölder's inequality,

$$
\begin{aligned}
\int_{\Omega} m(x) u(\lambda)^{2} d x & \leq \int_{\Omega} m^{+}(x) u(\lambda)^{2} d x \\
& \leq\left(\int_{\Omega} m^{+}(x)^{3} d x\right)^{1 / 3}\left(\int_{\Omega} u(\lambda)^{3} d x\right)^{2 / 3} \\
& =\left\|m^{+}\right\|_{L^{3}(\Omega)}\|u(\lambda)\|_{L^{3}(\Omega)}^{2} .
\end{aligned}
$$

Therefore, by using inequalities (A.3) and (A.4) we obtain that

$$
\begin{aligned}
\min _{\bar{\Omega}} h \cdot\|u(\lambda)\|_{L^{3}(\Omega)}^{3} & \leq \int_{\Omega} h(x) u(\lambda)^{3} d x \\
& \leq\left(1-\frac{\lambda_{1}(m)}{\lambda}\right) \int_{\Omega} m(x) u(\lambda)^{2} d x \\
& \leq\left(1-\frac{\lambda_{1}(m)}{\lambda}\right)\left\|m^{+}\right\|_{L^{3}(\Omega)}\|u(\lambda)\|_{L^{3}(\Omega)}^{2} .
\end{aligned}
$$

This proves that

$$
\|u(\lambda)\|_{L^{3}(\Omega)} \leq\left(1-\frac{\lambda_{1}(m)}{\lambda}\right)\left\|m^{+}\right\|_{L^{3}(\Omega)}\left(\frac{1}{\min _{\bar{\Omega}} h}\right),
$$


since $h(x)>0$ on $\bar{\Omega}$.

On the other hand, we have, by Hölder's inequality,

$$
\int_{\Omega} u(\lambda) d x \leq\left(\int_{\Omega} u(\lambda)^{3} d x\right)^{1 / 3}\left(\int_{\Omega} d x\right)^{2 / 3}=|\Omega|^{2 / 3} \cdot\|u(\lambda)\|_{L^{3}(\Omega)} .
$$

The desired decay estimate (1.10) follows by combining inequalities (A.6) and (A.5).

Similarly, in the Neumann case we can prove the decay estimate (7.3) in Remark 7.1, by making use of the variational formula due to Brown-Lin [4, Theorem 3.13].

\section{REFERENCES}

[1] R. A. Adams and J. J. F. Fournier, "Sobolev spaces", second edition, Academic Press, Amsterdam Heidelberg New York Oxford, 2003.

[2] H. Amann, Fixed point equations and nonlinear eigenvalue problems in ordered Banach spaces, SIAM Rev. 18 (1976), 620-709.

[3] H. Amann, Periodic solutions of semilinear parabolic equations, "Nonlinear analysis", (L. Cesari, R. Kannan and H. F. Weinberger, eds.), Academic Press, New York San Francisco London, 1978, pp. 1-29.

[4] K. J. Brown and S. S. Lin, On the existence of positive eigenfunctions for an eigenvalue problem with indefinite weight function, J. Math. Anal. Appl. 75 (1980), 112-120.

[5] R. S. Cantrell and C. Cosner, Diffusive logistic equations with indefinite weights: population models in disrupted environments, Proc. Roy. Soc. Edinburgh 112A (1989), 293-318.

[6] M. G. Crandall and P. H. Rabinowitz, Bifurcation from simple eigenvalues, J. Functional Analysis 8 (1971), 321-340.

[7] D. G. de Figueiredo, Positive solutions of semilinear elliptic problems, Lecture Notes in Mathematics, No. 957, Springer-Verlag, Berlin Heidelberg New York, 1982, pp. 34-87.

[8] K. Deimling, "Nonlinear functional analysis”, Springer-Verlag, Berlin Heidelberg New York Tokyo, 1985.

[9] J. M. Fraile, P. Koch Medina, J. López-Gómez and S. Merino, Elliptic eigenvalue problems and unbounded continua of positive solutions of a semilinear elliptic equation, J. Differential Equations 127 (1996), 295-319.

[10] J. García-Melián, R. Gómez-Reñasco, J. López-Gómez and J. C. Sabina de Lis, Pointwise growth and uniqueness of positive solutions for a class of sublinear elliptic problems where bifurcation from infinity occurs, Arch. Rational Mech. Anal. 145 (1998), 261-289.

[11] D. Gilbarg and N. S. Trudinger, "Elliptic partial differential equations of second order", 1998 edition, Springer-Verlag, New York Berlin Heidelberg Tokyo, 1998.

[12] P. Hess, "Periodic-parabolic boundary value problems and positivity", Pitman Research Notes in Mathematical Series, vol. 247, Longman Scientific \& Technical, Harlow, Essex, 1991.

[13] P. Hess and T. Kato, On some linear and nonlinear eigenvalue problems with an indefinite weight function, Comm. Partial Differential Equations 5 (1980), 999-1030.

[14] O. A. Ladyzhenskaya and N. N. Ural'tseva, "Linear and quasilinear elliptic equations", Academic Press, New York London Toronto Sydney San Francisco, 1968. 
[15] J. López-Gómez and J. C. Sabina de Lis, First variations of principal eigenvalues with respect to the domain and point-wise growth of positive solutions for problems where bifurcation from infinity occurs, J. Differential Equations 148 (1998), 47-64.

[16] A. Manes and A. M. Micheletti, Un'estensione della teoria variazionale classica degli autovalori per operatori ellitici del secondo ordine, Boll. Un. Mat. Ital. 7 (1973), 285-301.

[17] T. C. Ouyang, On the positive solutions of semilinear equations $\Delta u+\lambda u-h u^{p}=0$ on the compact manifolds, Trans. Amer. Math. Soc. 331 (1992), 503-527.

[18] C. V. Pao, "Nonlinear parabolic and elliptic equations", Plenum Press, New York London, 1992.

[19] P. H. Rabinowitz, Some aspects of nonlinear eigenvalue problems, Rocky Mountain J. Math. 3 (1973), 161-202.

[20] R. Redlinger, Über die $C^{2}$-Kompaktheit der Bahn von Lösungen semilinearer parabolischer Systeme, Proc. Roy. Soc. Edinburgh 93A (1982), 99-103.

[21] M. Reed and B. Simon, "Methods of modern mathematical physics IV", Academic Press, New York San Francisco London, 1978.

[22] D. H. Sattinger, "Topics in stability and bifurcation theory", Lecture Notes in Mathematics, No. 309, Springer-Verlag, New York Heidelberg Berlin, 1973.

[23] S. Senn, On a nonlinear elliptic eigenvalue problem with Neumann boundary conditions, with an application to population genetics, Comm. Partial Differential Equations 8 (1983), 1199-1228.

[24] S. Senn and P. Hess, On positive solutions of a linear elliptic eigenvalue problem with Neumann boundary conditions, Math. Ann. 258 (1982), 459-470.

[25] K. Taira, The Yamabe problem and nonlinear boundary value problems, J. Differential Equations 122 (1995), 316-372.

[26] K. Taira, Diffusive logistic equations in population dynamics, Adv. Differential Equations 7 (2002), 237-256.

[27] K. Yosida, "Functional analysis", sixth edition, Springer-Verlag, Berlin Heidelberg New York, 1980.

Kazuaki TAIRA is Professor of Mathematics at the University of Tsukuba, Japan, where he has taught since 1998. He received his Bachelor of Science (1969) degree from the University of Tokyo, Japan, and his Master of Science (1972) degree from Tokyo Institute of Technology, Japan, where he served as an Assistant between 1972-1978. He holds the Doctor of Science (1976) degree from the University of Tokyo, and the Doctorat d'Etat (1978) degree from Université de Paris-Sud, France, where he received a French Government Scholarship in 19761978. Dr. Taira was also a member of the Institute for Advanced Study, U. S. A., in 19801981. He was Associate Professor of the University of Tsukuba between 1981-1995, and Professor of Hiroshima University, Japan, between 1995-1998. His current research interests are in mathematical studies of Population Dynamics, Chemical Reaction and Elasticity by the extensive use of the ideas and techniques characteristic of the recent developments in the theory of partial differential equations.

Institute of Mathematics, University of Tsukuba, Tsukuba 305-8571, Japan

e-mail : taira@math.tsukuba.ac.jp 